Document downloaded from:

http://hdl.handle.net/10251/84418

This paper must be cited as:

Milián Sánchez, VM.; Mocholí Salcedo, A.; Milian, C.; Kolombet, V.; Verdú Martín, GJ. (2016). Anomalous effects on radiation detectors and capacitance measurements inside a modified Faraday cage. Nuclear Instruments and Methods in Physics Research Section A: Accelerators, Spectrometers, Detectors and Associated Equipment. 828:210-228. doi:10.1016/j.nima.2016.05.051.

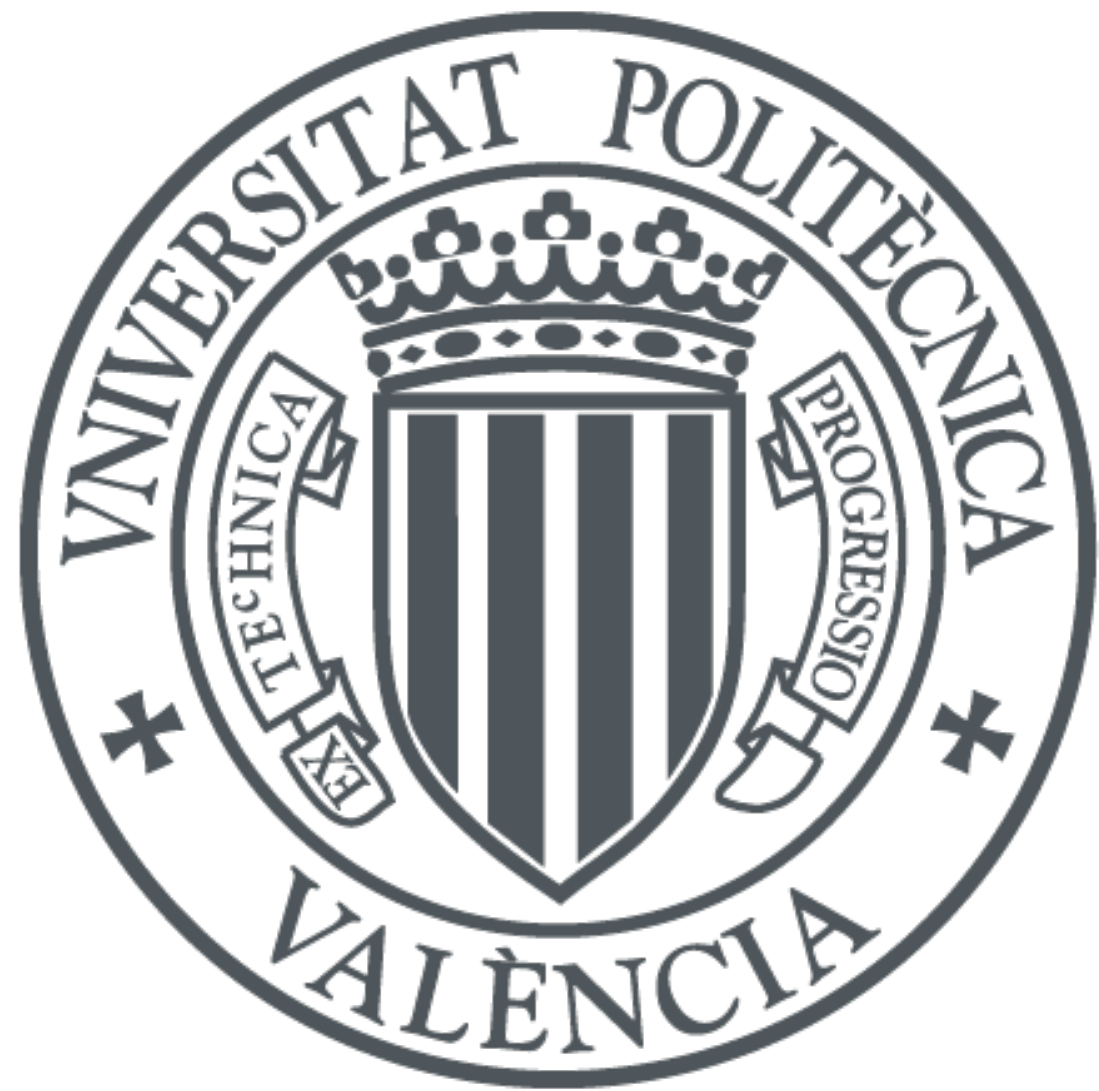

The final publication is available at

http://dx.doi.org/10.1016/j.nima.2016.05.051

Copyright Elsevier

Additional Information 


\title{
Anomalous effects on radiation detectors and capacitance measurements inside a modified Faraday cage
}

\author{
V. Milián-Sánchez ${ }^{\mathrm{a}, *}$, A. Mocholí-Salcedo ${ }^{\mathrm{b}}$, C. Milián ${ }^{\mathrm{c}}$, V.A. Kolombet ${ }^{\mathrm{d}}$, G. Verdú-Martín ${ }^{\mathrm{a}, e}$ \\ ${ }^{a}$ Institute for Industrial, Radiophysical and Environmental Safety, Universitat Politècnica de \\ València, Camino de Vera, s/n, Valencia, Spain \\ ${ }^{b}$ Traffic Control Systems Group, ITACA Institute, Universitat Politécnica de \\ Valencia, Camino de Vera, s/n, Valencia, Spain \\ ${ }^{c}$ Centre de Physique Théorique, CNRS, École Polytechnique, F-91128 Palaiseau, France \\ ${ }^{d}$ Institute of Theoretical and Experimental Biophysics, Russian Academy of Science, \\ Pushchino, Moscow Region, 142290 Russia \\ ${ }^{e}$ Chemical and Nuclear Engineering Department, Universitat Politécnica de \\ Valencia, Camino de Vera, s/n, Valencia, Spain
}

\begin{abstract}
We present the experimental results showing certain anomalies in the measurements performed inside a modified Faraday cage of decay rates of Ra-226, Tl-204 and Sr-90/I-90, of the gamma spectrum of a Cs-137 preparation, and of the capacitance of both a class-I multilayer ceramic capacitor and of the interconnection cable between the radiation detector and the scaler. Decay rates fluctuate significantly up to $5 \%$ of the initial value and differently depending on the type of nuclide, and the spectrum photopeak increases in $4.4 \%$. In the case of the capacitor, direct capacitance measurements at $100 \mathrm{~Hz}, 10 \mathrm{kHz}$ and $100 \mathrm{kHz}$ show variations up to $0.7 \%$, the most significant taking place at $100 \mathrm{~Hz}$. In the case of the interconnection cable, the capacitance varies up to $1 \%$. Dispersion also tends to increase inside the enclosure. However, the measured capacitance variations do no explain the observed variability in decay rates.
\end{abstract}

Keywords: capacitance variability; decay rates variability; modified Faraday cage; multichannel analyzer; multilayer ceramic capacitor; nuclides.

\section{Introduction}

Radioactive decay is usually considered to be a random process. However, the question of the nonrandom behavior in nuclear decay rates and if the decay constant can be somehow changed has long been of interest to the scientific community. Earlier studies, such as those of Rutherford and Geiger [1] and Curtis [2], provided evidence that those processes followed a Poisson distribution. However, it has been many years since non-expected results in radiation measurements have been reported and have been the object of numerous studies. As early as in 1924, Kutzner observed abnormal results in alpha decay of polonium [3]. He suggested the results were possibly caused by nuclear-nuclear interactions. Later, Anderson stated that the hypothesis of Kutzner could not be disregarded [4] and in a later paper he reported that $\gamma$ emissions detected following radioactive decay of Co-60 and Cs-137 nuclei deviated significantly form the theoretical (random) expectation [5]. In 1972, Emery discusses the macroscopic

\footnotetext{
*Corresponding author

E-mail address: vicmisan@iqn.upv.es (V. Milián-Sánchez), amocholi@eln.upv.es (A. Mocholí-Salcedo), carles.milian@cpht.polytechnique.fr (C. Milián), kolombet@iteb.ru (V.A. Kolombet), gverdu@iqn.upv.es (G. Verdú-Martín).
} 
ways of changing the rates of electron capture and internal conversion [6]. In 1977, Dostal et al. provided a review of the fundamental principles used to explain the decay rates variations produced by chemical or physical means [7]. Other researchers, like Norman et al. [8], measured the electron-capture decay rate of Be-7 and of K-40 (implanted into different hosts), with $0.38 \%$ and non-detectable decay variation respectively.

Recently, this question has gained renewed interest due to the evidence for a correlation between nuclear decay half-lives and Earth-Sun distance and also by the observation of a dip in the decay rate for Mn-54 which coincided with the 2006 December 13 solar flare $[9,10]$. One of the most recent papers in this field is the one of Sturrock et al. about the anomalous results in an analysis of beta-decay rates of several nuclides acquired at the Physikalisch-Technische Bundesanstalt from 1990 to 1995 [11]. The authors state that the presented results about oscillations and periodicities in decay rates were compatible with a solar influence; they do not appear to be compatible with an experimental or environmental impact, and it is possible that Ba-133 measurements are also subject to a non-solar (possibly cosmic) factor $[11,12]$. Nowadays, there is as yet no accepted theory to explain beta-decay variability, but according to Fischbach et al. the hypothesis proposed by Falkenberg [13] that beta-decay may be influenced by neutrinos should be taken into account (a similar hypothesis has been proposed by Parkhomov [14]). Also, to try to explain the results, they refer to the possible variations in the magnitudes of fundamental parameters, such as the fine structure constant and the electron-to-proton mass ratio [9]. Furthermore, and based on a detailed description of the empirical evidence for the variability and periodicity of decay in a range of nuclides, Pons et al. provide a candidate novel theoretical contribution for the explanation of that variability [15].

Another approach regarding fluctuations in decay processes has been developed by Shnoll et al. In the 1960s they suggested that the scatter in experimental measurements (of noise processes) was not entirely random, this effect being caused by some general unknown cosmophysical (cosmogonical) factor(s) [16]. This can be seen through the comparison of the histograms obtained from those measurements in a series of experiments: whether the processes consist in the rate of a chemical reaction, the fluctuation noise of the rate of dark current measured in a photomultiplier [17], the radioactive decay or any other process generating a random signal, a sequence of histograms can be obtained from those signals by dividing the time series in short non-overlapping consecutive intervals [18]. Then, when analyzing and comparing the corresponding successive histograms, one can observe the non-randomness of the obtained measurements in a set of characteristics $[19,20,21]$. One of these characteristics is the "near-zone-effect" (NZE): this means that the relative number of similar histograms is reproduced with high probability in time intervals which are consecutive to each other (this effect can be correlated with some solar-geophysical indices [22]). Another characteristic is that the similarity of histograms tends to repeat itself at definite intervals close to the length of an Earth day and year (i.e., there exist a periodicity in the fine structure of histograms).

Aside from Fichbach, Sturrock and Shnoll, other researchers have also found, over the course of many years, influences of geo-cosmic factors on chemical, physical and biological processes, [23, 24, 25, 26]. Even as early as the first part of the past century, researchers like Piccardi, Mosseti, Capel-Boute and Bortels [27], became aware of the effect of external spatial agents (and particularly of the solar activity) on chemical, physical-chemical and biological processes [23]. So, in 1951 Piccardi and coworkers begun to verify that the non-reproducibility of experimental results on different physical-chemical as well as biological systems was related to Earth movements (around the Sun and along its path through the Galaxy) and to solar activity. In order to try to isolate the systems under study from external components (electromagnetic radiation) he used metallic screens, both in form of a Faraday cage or simply as a 
metallic cover, thus executing his chemical test under those conditions (the test consists in the hydrolysis of bismuth chloride in presence of acid and production of bismuth oxychloride). One of the conclusions was that those experiments were affected in one way or another depending on the nature of the metal screen, one of the most important characteristics being its specific electrical conductance [27].

The results of those tests (test $\mathrm{D}$ inside, and test $\mathrm{F}$ outside the cage), showed that, the smaller the solar activity was, the more different the results were. Besides, tests D (performed by the 1950s), revealed an annual minimum during the months of March, when the Earth is directed approximately towards the galactic center at maximum speed; in other words, test D seemed to reveal the effects of the helical motion of the Earth in the Galaxy: this was Piccardi's solar hypothesis. Later, a shift in annual variation of tests D over the years was observed, which was related to the influence of other cosmic objects [28]. Piccardi's tests have been thoroughly studied along the years and his results have been analyzed and confirmed by many researchers [29]. Similarly to the Shnoll effect, it was also interpreted from a relativistic point of view [30].

Other works with metallic enclosures which also yield anomalous results were performed in the field of biology and biophysics. So, by the 1930s, Reich also found specific biological reactions when using a modified Faraday cage (MFC). This structure consisted in a multilayer- metallic enclosure in which each side was made from alternating layers of metal and organic substance [31].

Over the course of many years, dissertations and experiments with Reich's enclosure have been performed [32,33], and recently, experiments about the influence of this MFC on biological systems and about its influence on the water structure by significantly increasing its structured fraction, have been presented [34]. But aside from the many biological and physical effects Reich described, one of them refers to Ra-226 decay rates measurements which seemed to show anomalous fluctuations [31].

Thus, based upon the results obtained by Piccardi and Reich with metallic screens, upon the experimental results presented by Fischbach, Sturrock and Shnoll, and recently by Farshchuck et al. [34], following questions are raised.

a) Whether it can be statistically confirmed that a (multilayer-) metallic enclosure can also affect radiation measurements obtained with a Geiger-Müller (GM) counter tube, specifically, (average values of) background counts and decay rates from different radioactive sources, (as well as other); the same question can be raised regarding the spectrum obtained with a multichannel analyzer (MCA).

b) Whether some specific effect/trend can be observed along a 24 hours or longer periods, or along different months.

c) Besides, since radioactivity measurements obtained with a GM detector and a MCA are the first objects of this investigation, the question was also raised on whether possible anomalies could be caused by some effect on the circuitry of the detectors. Since the electronics of these devices include a combination of resistors $\mathrm{R}$ and capacitors $\mathrm{C}$, and since the pulses shape depends on the RC value [35], it has been investigated: 1) the response of a low-pass RC filter inside such structure, and 2) the stability of capacitors' impedance inside it.

In this paper we show that all the performed measurements, namely, background counts, decay rates of radioactive samples, the spectrum of a nuclide as well as the low-pass filter time constant and a capacitor's capacitance, change and/or oscillate significantly (although with variable intensities along the time) when taken inside the MFC. It is worth mentioning that whereas the absolute values of the 
measurements are not always reproducible in the sense of reference [27], the fluctuations and jumps in data are systematically detected. In Section II we present the results along with the discussion and in Section III the summary and conclusions are outlined. In the Appendix, materials and methods are described.

\section{Results and discussion}

In what follows, the results obtained from the comparison of measurements both outside and inside the MFC are shown. As mentioned in the Appendix, simple statistical analysis is the tool to compare the corresponding data sets. Unless otherwise specified, all analyses are performed at the $99 \%$ confidence level. Also, the results are often presented as the mean along with the confidence interval of the mean, i.e., as $\pm 2 \sigma_{\mathrm{m}}$.

\subsection{Background counts}

The functioning of the GM counter tube was firstly checked. This analysis showed a plateau slope in accordance with specifications, i.e. $\leq 0.04 \% / \mathrm{V}$. On the other hand, two data groups of background measurements (denoted by OUT-1 and OUT-2), taken outside the box during two separated periods of about four and two hours respectively (at time intervals of $1 \mathrm{~min}$ ) fit a Poisson distribution (and are compared later in Fig. 1a). These tests ensure that the GM counter tube works correctly.

The stability of the measurements in a further test outside the box over a 19 hours period (OUT-3) was also verified by means of an ANOVA analysis (not represented). With this purpose, counts were recorded successively each minute and thus, the data recorded each hour were pooled in nineteen groups of sixty data each one. Since all these data groups fit quite well a normal distribution and variances were statistically equal, the comparison between the groups could be performed. The numerical results for Fratio and $\mathrm{P}$-value are $\mathrm{F}=0.64, \mathrm{P}=0.8792$ respectively. Therefore, all the nineteen groups had the same mean. The grand average value was $16.53 \mathrm{cpm},(0.27 \mathrm{cps})$ and the standard deviation $\sigma=0.43 \mathrm{cpm}$ (0.007 cps).

It is noteworthy that a subsequent test outside the box (OUT-4) was repeated during 26 hours about nine months after the onset of the experiments and yielded a similar result (not represented): neither significant difference amongst the means nor significant difference amongst the variances of the data groups could be observed. In this case, the grand average was $16.20 \mathrm{cpm}(0.27 \mathrm{cps})$ and the standard deviation $0.55 \mathrm{cpm}(0.009 \mathrm{cps})$. Thus, it could be assumed that a similar result would be obtained for longer measurement periods. Moreover, the comparison of the two data sets OUT-3 and OUT-4 (using a t-test) yields that both the means and the standard deviations are equal.

After the confirmation of stability outside the enclosure, the next step consisted in comparing results of measurements taken both outside and inside the MFC. For this purpose tests inside the box followed in different ways. Firstly, measurements were taken discontinuously over several days and at different hours; then, results were compared with the two previous tests outside the box (OUT-1 and OUT-2, as shown in Fig. 1a). Secondly, measurements were taken continuously over a number of days (divided in four periods) but alternating the GM location outside and inside the shield to search for differences (Fig. 1b). These comparisons are described in what follows. 


\subsubsection{Background counts comparison. Some discontinuous measurements}

As mentioned before, Fig. 1a shows some background measurements comparison both outside and inside the MFC: since these first data groups fit a Poisson distribution, the comparison was performed by means of the Kruskal-Wallis test to compare medians (using a Box-and-Whisker plot). The first two tests, OUT-1 and OUT-2, do not show a statistical significant difference amongst the medians; but the following four tests on the inside, IN-1 to IN-4 (also taken during periods of similar length of about three hours, at $1 \mathrm{~min}$ intervals) differ significantly from previous ones $(12 \mathrm{cpm}$ outside and $18 \mathrm{cpm}$ inside the shield), whereas they do not differ from each other. All variances are equal and the number of outliers tended to be higher inside the box. These results were obtained in 2014 June and 2014 July.

Beyond the differences in median values, skewness and kurtosis also seemed to show noticeable increments inside the cage (for instance, kurtosis in test IN-3 being 13.4 whereas in all other cases it was in the range \pm 2 ). Besides, although the variance values do not show a statistically significant increase (which can be seen by using the median based Levene test for variances comparisons [36]), they showed an upward trend with time: this possible result deserves more investigations to check if a significant change (and/or fluctuations) in variance could take place after a period of time long enough (this possibility was taken into account in the next cases).

Although GM counters are very stable and robust detection systems [10, 37, 38] previous results show that counts at that time inside this MFC were higher and more dispersed than in the outside, which is contrary to what could be expected (the stability of GM counters has been discussed in the Appendix). This behavior will also be observed later (section 2.2), when measuring decay rates of radioactive samples.

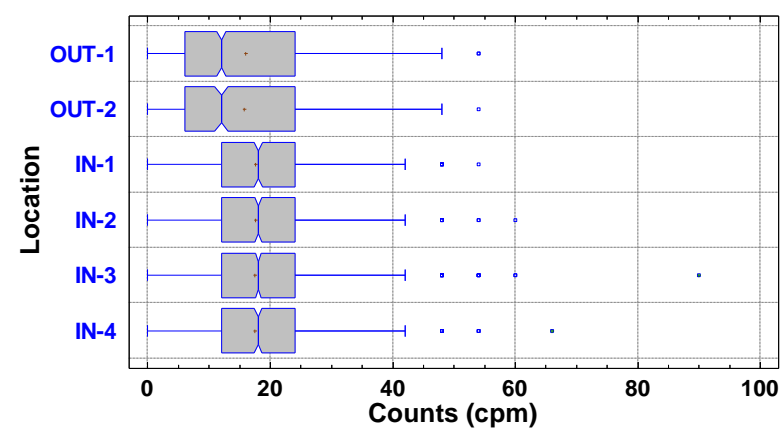

(a)

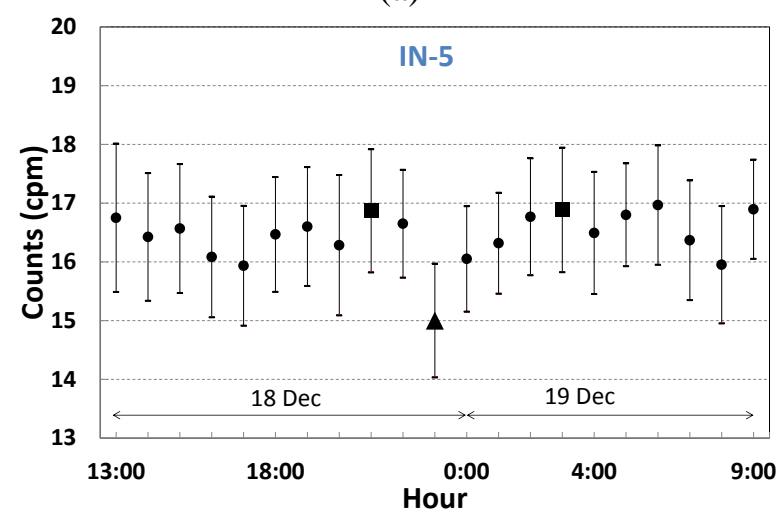

(c)

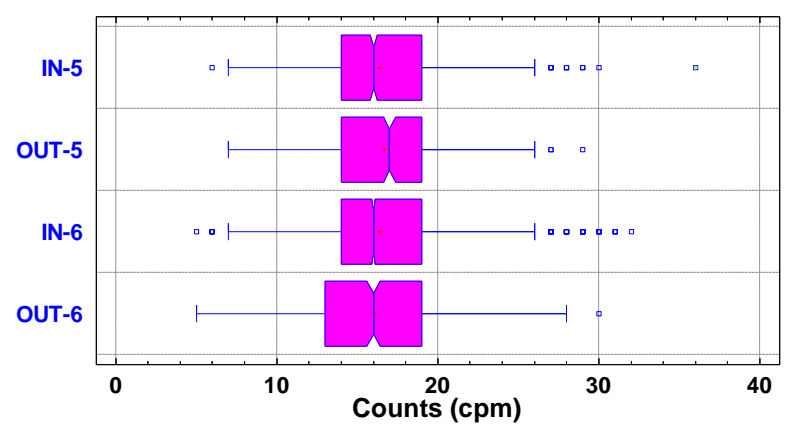

(b)

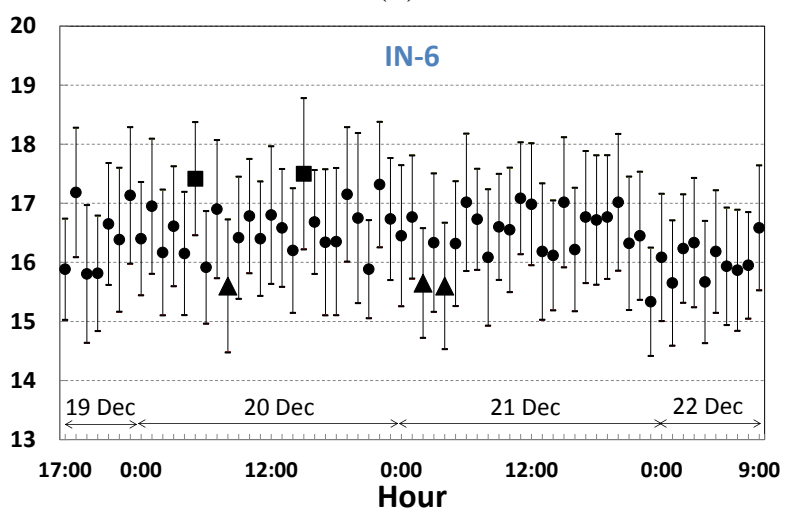

(d) 
Fig. 1 Background counts comparison and analysis. a) Box-and-Whisker plot to compare median values of background counts measurements both outside (OUT) and inside (IN) the MFC. Data were taken on June and July 2014. Counting rates increase from $12 \mathrm{cpm}$ to $18 \mathrm{cpm}$. Outliers tend to increase inside the shield. b) Alternating background counts comparison during four periods in 2014 December: two periods inside the MFC, IN-5, IN-6, and two periods on the outside, OUT-5, OUT-6, all of which have the same medians and variances except for skewness, which tends to increase inside the cage. c) Data groups constituting period IN-5 of subfigure-b). d) Data groups constituting period IN-6 of subfigure b). Some of the means in c) and d) that are different have been marked with a square (high values) and with a triangle (low values). All times and dates in all Figures are reported in UTC.

\subsubsection{Continuous and alternating background counts}

The next step was to perform continuous and alternating tests inside and outside the box for background counts measurements some time later (Fig. 1b). This was carried out during four additional periods in 2014 December. Counts were recorded each minute and pooled in groups of sixty data per group (one group each our). These successive periods and time span were: IN-5, from 2014 December 18 at 13:00 to 2014 December 19 at 09:00; OUT-5, ranged from 2014 December 19 at 10:00 to 2014 December 19 at 16:00; IN-6, from 2014 December 19 at 17:00 to 2014 December 22 at 09:00, and OUT6, from 2014 December 22 at 10:00 to 2014 December 22 at 18:00.

Contrary to what shown in Fig. 1a, the comparison of those four periods of Fig. 1b showed that there was no difference amongst the medians: this time, variances were equal and the skewness of periods IN-5 and IN-6 are 3.5 and 7.3 respectively; kurtosis of IN-5 is 2.6. Thus, it raises the question on whether the emergence of such differences could show some seasonality. This possibility was taken into account along the whole experimental period.

On the other hand, when comparing to each other (by means of a t-test) the data groups which constitute OUT-5 and OUT- 6 in Fig. 1b, no difference amongst the means of the groups belonging to those periods appeared (as with the longer data groups in OUT-3 and OUT-4). Another set of data, OUT7 (not represented), taken during 68 hours in 2015 July 15, showed the same stable results. However, when comparing to each other the groups constituting the periods IN-5 and IN-6 (of subfigure b) one can observe groups with different means. This is shown in Fig. 1c (for period IN-5) and in Fig. 1d (for period IN-6), where the crosses and triangles mark some of the groups with different means (confidence intervals for the means are represented). They generally are different at the $95 \%$ confidence level although some pairs are different at the $99 \%$ confidence level.

Other observations can be drawn from the previous tests (Fig. 1c and Fig. 1d). Firstly, it seems that fluctuations with significant changes in mean (median) values (and eventually in variances, skewness and kurtosis), are usually noticeable, not immediately after the onset of the measurements inside the box, but after a certain amount of time, in contrast to the stationarity observed during the tests outside the box. Also, it could be due to chance, but some maxima and minima in cpm can be observed close to 00:00 or 12:00 hours. All this will be observed again in the next measurements.

Thus, from the described experiments, the question arises on whether the increase in background counts along with the variations in skewness and kurtosis is the result of an increase (and fluctuations) in spurious counts, and/or by perturbations of the counter circuitry, (basically composed by resistors and capacitors). In that case, this would raise the additional question on the reason of the appearance of such perturbations inside the MFC, and about a model that could explain them. 
Various physical mechanisms lead to the production of after-pulses [35, 39, 40]. One of them consists in the production of low-energy electrons at the cathode surface through the photoelectric effect. The latter, in turn, is caused by photons coming from excited atoms produced in the avalanche. Other seemingly not fully understood mechanisms are described in the literature [41]. Some of them are field emission of electrons at the cathode wall or metastable states of the counter gas. Assuming that there are no trivial errors in the presented experiments, one could ask about the reasons for such mechanisms to be enhanced inside the MFC. For now, it can only be said that all happens as if electrons were more ready to cross the potential barrier in the cathode metal surface, and as if the metastable states of the gas could discharge their excess of energy more easily.

In what follows, we present the decay rate results obtained with the radioactive preparations. Later on, changes in the spectrum of a Cs-137 source are briefly outlined.

\subsection{Decay rates of several radioactive samples}

The next two paragraphs refer to measurements which were performed with different preparations and in different ways. Firstly, data were taken during short and discontinuous periods using two preparations of Ra-226 and Tl-204. Later, continuous decay rates were measured during longer periods using an additional Sr-90/Y-90 source. In this way: a) the repeatability of results can be seen and b), the latter way of measurements allows the observation of variations which otherwise would have remained unnoticed.

\subsubsection{Short and discontinuous measurements of Ra-226 and Tl-204 decay rates}

As regards the decay rate measurements of radioactive sources, Fig. 2 shows the results of the first five and eight measurements using the Ra-226 and Tl-204 preparations respectively, both outside and inside the box: they were taken in a discontinuous way during short periods of time (two/three hours in average each day) along several days (in June and July 2014).

\section{a) $\mathrm{Ra}-226$}

In Fig. 2a, the mean value of the first test outside the box (on 2014 June 23) yields about $17850 \mathrm{cpm}$. It differs from the means of the three following three tests, which in turn are about $600 \mathrm{cpm}$ higher, (i.e., about 3\%) and statistically equal to each other (LSD intervals overlap). But the fourth measurement (on 2014 July 31, which was taken with both the lead container and the scaler inside the box), drops significantly and only differs in $300 \mathrm{cpm}$ or $1.9 \%$ from the first test. 


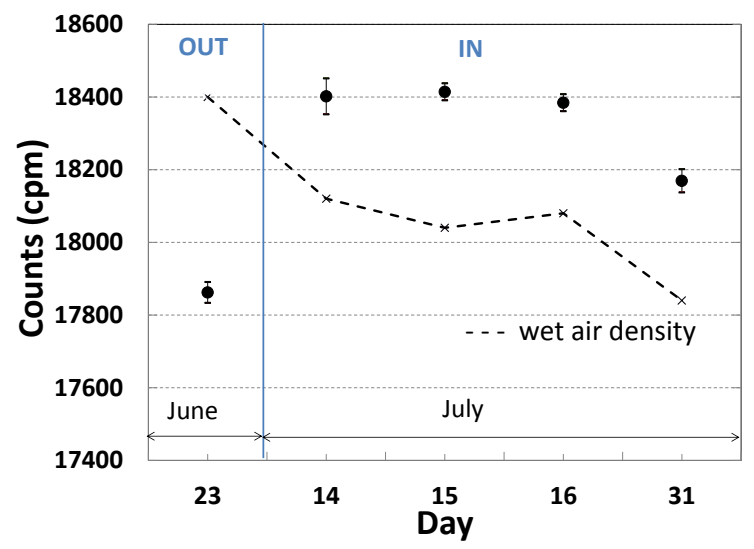

(a)

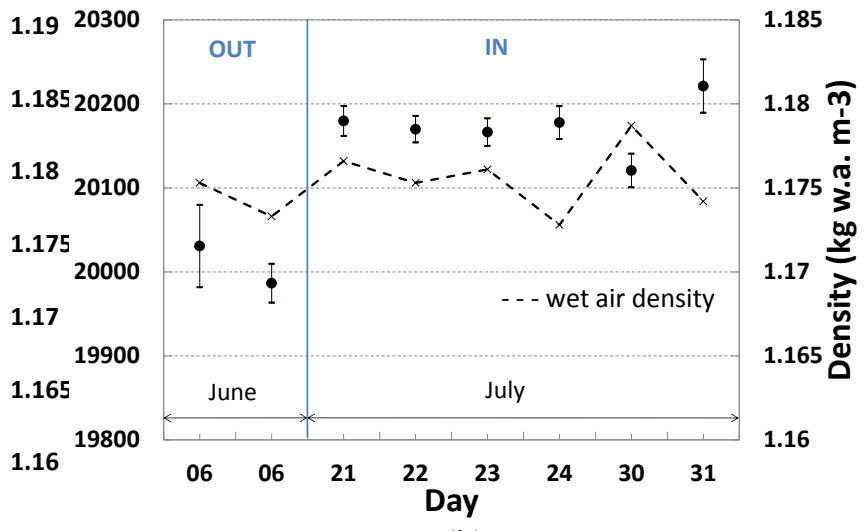

(b)

Fig. 2 Some short and discontinuous decay rates measurements both outside and inside the box. a) Ra226 decay rate outside the box (OUT, on 2014 June 23), and three measurements inside it (IN, on 2014 July), which are about $3 \%$ higher. The fourth measurement was taken with both the lead container and the scaler inside the enclosure (on 2014 July 31), and differs about $1.9 \%$ from the first measurement. The dashed line is the wet air density: changes in decay rates do not correspond to changes in air density and in ambient temperature. b) Two different Tl-204 decay rates measurements (outside the box) on 2014 June 6 followed by six more (inside) on 2014 July. Results inside the MFC increased about $0.8 \%$. Fluctuations appeared in the two last measurements when all the instruments were placed inside the cage. The dashed line is the wet air density. There is no correlation between both series of values and measurements are not determined by ambient temperature either.

On the other hand (as when measuring background counts), as time passes, some statistics tend to deviate from their initial values; so, on this occasion skewness and kurtosis of data taken on 2014 July 31 took the values 2.3 , i.e., they came slightly outside the limits corresponding to a normal distribution (i.e., $-2 /+2)$. Also, as in previous Figures, the number of outliers increased inside the MFC.

As mentioned in section A.1, ambient temperature and (wet) air density have to be examined to ensure that they are not factors which explain the observed changes in decay rates. Air density must be taken into account because it determines the more or less loss of energy of beta particles when crossing the gap between source and detector's mica window. Wet air density $\rho$ (in $\mathrm{kg} \mathrm{w} . \mathrm{a} . / \mathrm{m}^{3}$ ) can be obtained from text books and is given by [42]:

$$
\rho=\frac{1+\theta}{v_{S}}
$$

Where $\theta$ is the absolute humidity and $v_{s}$ is the wet air specific volume, which in turn depends on the relative humidity (RH). Values of $\rho$ obtained from Eq. (1) are shown in Fig. 2a: by simple inspection one can see that that mean value on test on July 31 should be higher than the other following three values on the inside: therefore, no dependence from this environmental factor can be deduced. Another procedure to calculate this density is given in Ref. [43, 44]: as shown in Fig. 3 of Ref. [10], air density decreases when temperature increases and when RH increases.

In regard to ambient temperature, maximum temperature oscillation was less than four degrees centigrade which does not justify the observed variations in decay measurements. 


\section{b) $\mathrm{Tl}-204$}

In Fig. $2 b$ an ANOVA analysis for the measurements with Tl-204 shows that decay rates in the first two tests on the outside (on the same day 2014 June 06 ) are only about $0.8 \%$ (or $180 \mathrm{cpm}$ ) lower than in the following four tests on the inside (from 2014 July 21 to July 24). Variances are not different. It is noteworthy that the observed increase in cpm was much lower than in the case of radium.

Afterwards, as in the case with radium, the whole system was placed inside the box (i.e., the lead container together with the scaler): here again the first reaction was a decrease in cps (on 2014 July 30), but a subsequent test showed an increase in decay rate (on July 31). Aside, outliers also tended to increase.

The values of skewness of the measurements taken on July 22, 23, and 30 are about 3.2, thus slightly outside the limits for a normal distribution (a median analysis also yields that medians are different).

As in the previous case, Fig. $2 b$ also presents wet air density for all measurements periods. Here again, there is no model which correlates both series of data. Moreover, from June 06 to July 21 there was a drop of less than two degrees centigrade, which does not explain the increase in cpm either.

\subsubsection{Longer and continuous decay rates measurements of Ra-226, Tl-204 and Sr-90/Y-90}

Previous tests were performed discontinuously during short intervals. The next steps consisted in the realization of longer and continuous measurements in different ways and with three different radioactive sources: the two previous ones and an additional of Sr-90/Y-90.

\subsubsection{Measurements with Ra-226}

a) Ra-226 decay rate with GM4 inside the box

In the next experiment, one can see the evolution of decay rate measurements taken consecutively during 91 hours (Fig. 3a). In this case a different counter tube GM4 was used; this tube has the same characteristics except for that the lead container has slightly thinner walls than the former one. As before, each point in Fig. 3a represents the mean of 60 measurements of one minute duration and each side of the bars represent two times the error of the mean $\sigma_{\mathrm{m}}$. Almost all those individual measurements fit a normal distribution as well as the complete set of data, and there is not a statistically significant difference amongst the standard deviations.

This experiment began on 2014 December 5 at 13:00. For some reason, a first transient-like ascending ramp started after about two hours of placing GM4 inside the shield, and the first measurement which differed from the two firsts ones appeared five hours after the onset of the experiment (marked with a triangle). These two points differ in more than $4 \sigma_{\mathrm{m}}$. A t-test gives obviously a significant difference between those means.

The first ascending ramp (transient) developed during nine hours and was followed by another descending transient. It might also be by chance, but the first peak appears coincidentally around 00:00 (similarly as in Fig. 1c) and represents an increment of $0.8 \%$, (or $\left.>4 \sigma_{\mathrm{m}}\right)$ as in the case of Tl-204 in Fig. $2 \mathrm{~b}$, but it was a quite lower increase in comparison with Fig. 2a (decay rates of Ra-226 inside GM1 and on 2014 June and July). After the first unexpected oscillation, more (equally abnormal) slight fluctuations 
followed, along with a gradual increase in cpm throughout the hours, and a last jump was observed during the last 12 hours. The highest cpm values were reached after about 84 hours from the beginning and were about $1.1 \%$ higher than the initial two means and about $12 \sigma_{\mathrm{m}}$. However, this increment was still much lower than the one observed in Fig. 2a. A longer observation time in similar conditions would be necessary to verify if further ascending jumps could take place, but again the question arises about a possible seasonal effect.

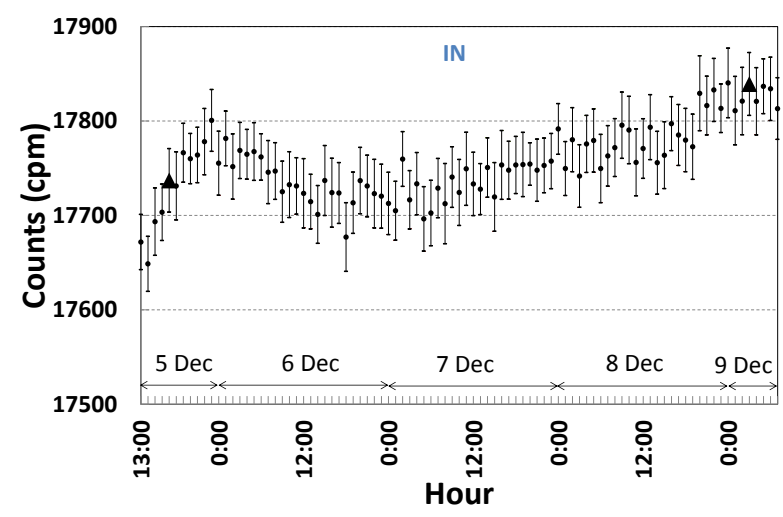

(a)

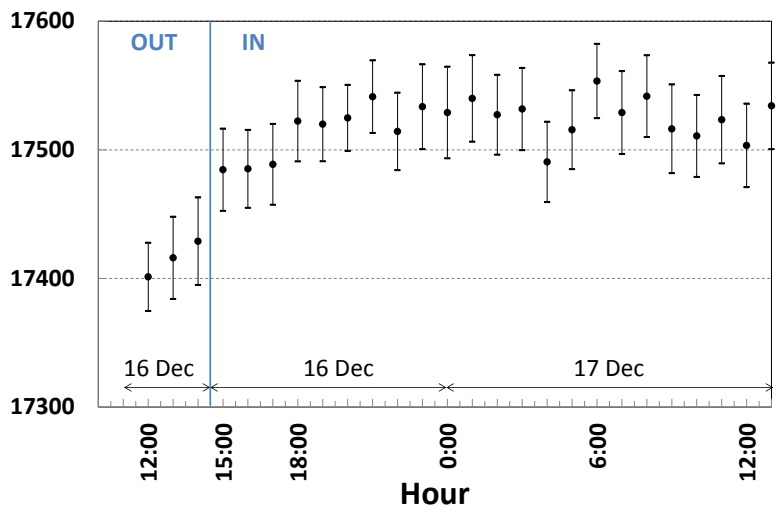

(b)

Fig. 3 Measurements taken with a second counter GM4 using Ra-226 on 2014 December. a) Continuous decay rate measurements inside the MFC during 91 hours, starting on 2014 December 5 at 13:00. A first strong oscillation develops with a first increase of $0.8 \%$ (or $>4 \sigma_{\mathrm{m}}$ ), and a subsequent ascending ramp until it reaches a $1.1 \%$ increase in cpm (or $12 \sigma_{\mathrm{m}}$ ). This increase cannot be explained by environmental factors. b) Transient after placing the source inside the box one week later; it is similar to the transient in Fig. 3a. The cpm at the starting points in a) and b) are different.

As regards the possible influence of environmental factors, (outdoors) temperature dropped from 17.9 ${ }^{\circ} \mathrm{C}$ on the onset of the test to $10^{\circ} \mathrm{C}$ at the point where the first maximum in cpm readings was reached. Therefore, without that temperature variation, actually recorded cpm would have been up to $0.36 \%$ higher (see Appendix). Moreover, since air density also increased from about $1.215 \mathrm{~kg} \cdot \mathrm{m}^{-3}$ to 1.250 $\mathrm{kg} \cdot \mathrm{m}^{-3}$, true readings would have been even higher. A similar reasoning can be made regarding the maximum located on 2014 December 9 at 04:00 (marked with a triangle), where temperature is $12.4{ }^{\circ} \mathrm{C}$ and air density is $1.2402 \mathrm{~kg} \cdot \mathrm{m}^{-3}$.

b) More measurements of Ra-226 decay rate with GM4 and subsequent background counts.

This experiment is similar to the previous one and was performed one week later with the same counter GM4, but only during 26 hours (results in Fig. 3b). The objectives were: 1) to check once more that decay rates measurements increases and/or fluctuates inside the MFC, 2) that this increase develops in a similar way as seen in Fig. 3a, and 3) to check the values of background counts, and specifically, to check and to look for a possible remnant effect in the recorded background counts after removing the Ra226 source from inside the lead container but leaving it inside the MFC. The first point (outside the box) is $17400 \mathrm{cpm}$, or $1.4 \%$ below the first point in Fig. 3a (with no apparent reason), and about $2.5 \%$ (or $24 \sigma_{\mathrm{m}}$ ) lower than the highest value in that Figure. The approximated confidence intervals show again the statistically significant differences between means of tests performed outside and inside the MFC.

The first ascending ramp that took place in this case (Fig. 3b) is similar to the one in Fig. 3a. Also this time the upward trend started about two hours after placing the GM4 inside the box and developed during 
the following seven hours: the highest increase in counts was again of about $0.8 \%$, which had been repeatedly observed (see Fig. 2a and Fig. 2b), but did not reach similar cpm values as those in Fig. 3a (possibly due to a much shorter measurement interval). Afterwards no strong oscillations were noticed, either.

In regard to environmental factors, air temperature and density were $17.1{ }^{\circ} \mathrm{C}$ and $1.2191 \mathrm{~kg} \cdot \mathrm{m}^{-3}$, respectively, at the onset of the test on 2014 December 16 at 13:00, whereas nine hours later (at 22:00) when the source was inside the box, those parameters were $13.6^{\circ} \mathrm{C}$ and $1.2347 \mathrm{~kg} \cdot \mathrm{m}^{-3}$. Once more, these changes do not correspond to the cpm observed variations.

On the other hand, after extracting the source from GM4, but leaving the container inside box, the background counts did not show any strong and noticeable remnant effect. Only slight differences between background results inside and outside the box were noticed (not represented). Again, the question arises on whether this is caused by a tendency of spurious pulses to increase when measuring inside the MFC.

In what follows all measurements were performed with the GM1 counter tube.

c) Ra-226 decay rate alternating measurements on 2015 January

Previous experiments in Fig. $3 \mathrm{a}$ and Fig. $3 \mathrm{~b}$ showed the instability and the responses appearing in measurements when the preparation is placed and kept inside the MFC for a longer time than in Fig. 2a and Fig. 2b. The next step consisted in observing the measurements results when inserting and removing repeatedly the GM1 counter from the enclosure (the source was left inside or outside the enclosure for hours). Particularly, it was intended to check again the existence of the aforementioned ups and downs in cpm and to analyze the counts evolution outside the MFC after the unstable counting rates were registered inside it. Besides, although it could be assumed that tests on the outside would be stable overtime with no statistically significant oscillations (like the first points in Fig. 2b, Fig. 3a, and Fig. 3b and also subsequently, in the case of $\mathrm{Sr}-90 / \mathrm{Y}-90)$, it was also thought to check once more this assumption about our counting system. The results are presented in Fig. 4a, where one can distinguish different groups for the measurements taken on 2015 January. It is worth noting that the initial cpm values (outside) were close to the last values in Fig. 2a (inside).

Measurements results in Fig. 4a are similar to those in Fig. 2 and Fig. 3 in the sense that they show the already known and more or less intense expected fluctuations (during the 31 hours that the source was inside the shield). The first three means on the outside (starting on 2015 January 07 at 13:00), were equal. But after several hours of having inserted the source inside the box (at 16:00), they were followed by a slight increase of $0.5 \%$ (less than in Fig. 2a and Fig. 3a), with a first peak on January 7 at 19:00, and by a subsequent oscillation (in a seemingly unpredictable manner) of the recorded means (a small peak appeared nearby 00:00 on January 8 and a second peak on January 8 at 13:00, nearby solar noon). Then, after extracting the GM1 from the shield (on 2015 January 8 at 16:00), an abrupt drop in cpm took place. This was followed by 18 hours on the outside (until 2015 January 9 at 09:00) with no noticeable significant oscillations of the means. And as usual, some data groups in the subsequent period on the inside (on January 9 at 11:00 and at 14:00 showed different means (and medians) and variances: test $F$ for variances comparison yields $\mathrm{F}=1.86458$ and $\mathrm{P}=0.0180729$, lower than 0.05 ; aside, $\mathrm{P}$-value $=$ 0.0122917 for medians comparison). 
The oscillations described in the previous paragraph reach sometimes values that differ significantly from the means on the outside, although in this experiment the difference between the most significantly different values is of $0.8 \%$, which is similar to the observed variations in Fig. 2b, Fig. 3a and Fig. 3b (considering that measurements were taken at different months): however, these small differences in percentage are about $4 \sigma_{\mathrm{m}}$ or even $6 \sigma_{\mathrm{m}}$. Aside, the highest value is almost $3 \%$ higher than the first measurement in Fig. 2a (about $17880 \mathrm{cpm}$ ), although it dropped later (see next subsection).

The recurrent questions arises on whether the intensity of the effect is dependent on the season (or on the month), and in that case, which would be the reason of this effect. The response to the former question requires more observations along several years. Regarding the latter question, one possible explanation could be the appearance of fluctuations in detector's dead time which in turn would allow more or less pulses to be detected. But this would imply fluctuations in the value of the measuring circuit time constant.

d) More Ra-226 measurements on 2015 February and March

Next test with Ra-226 inside the box was undertook from 2015 February 27 to 2015 to March 2, (Fig. $4 b)$. The confidence intervals for the means are given by $\pm 2 \sigma_{\mathrm{m}}$. It is noteworthy that the initial decay rates (about $17900 \mathrm{cpm}$ ) were similar to the values at the onset of the experiments in 2014 June and July, i.e., $1.8 \%$ lower than at the end of Fig. $4 \mathrm{a}(18250 \mathrm{cpm})$.

Furthermore, measurements behaved similarly to what seen in previous tests with the same source, although this time two successive peaks appeared during the first ten hours. The initial increase in cpm is of less intensity and (again) only an increase of about $0.8 \%$ was reached. After the two first oscillations a long period of about 34 hours of relative stability followed at about the same initial counting rate (17880 cpm) although some different means appeared (marked in the Figure).

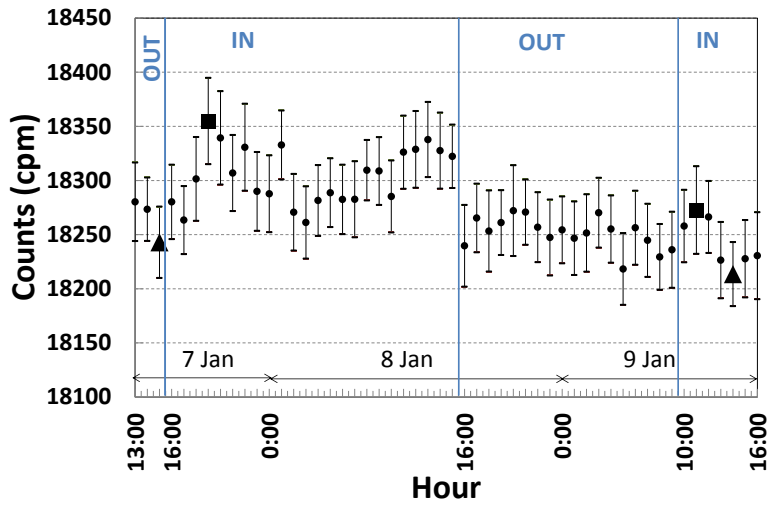

(a)

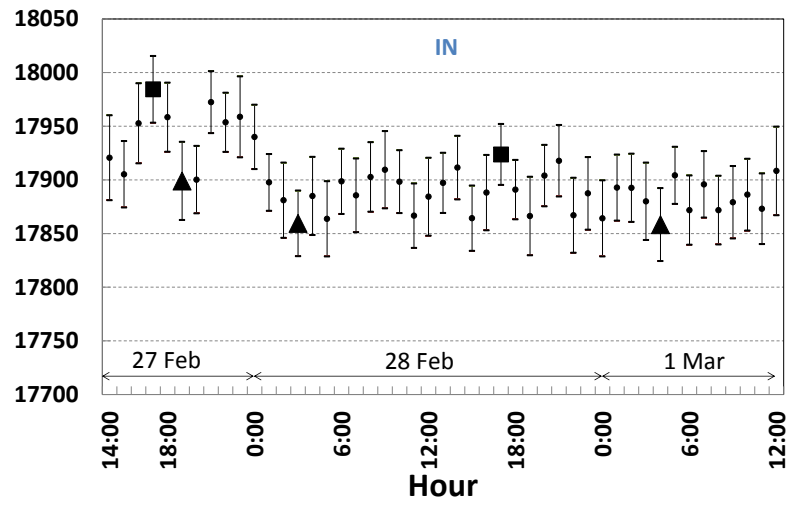

(b) 


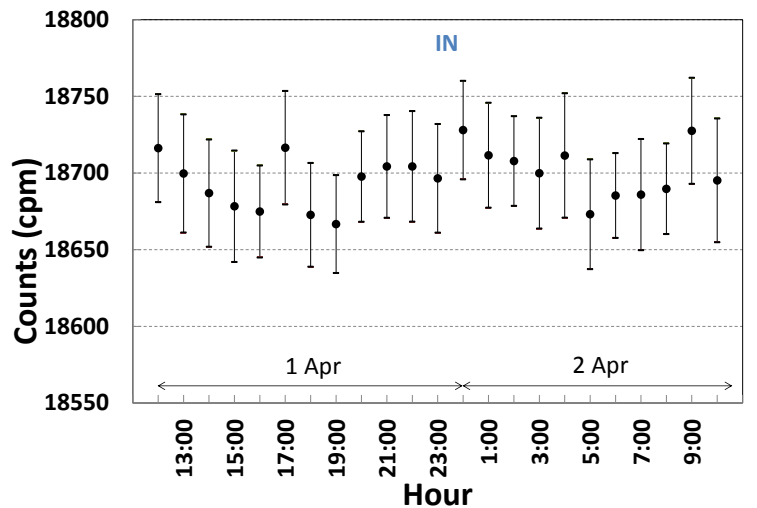

(c)

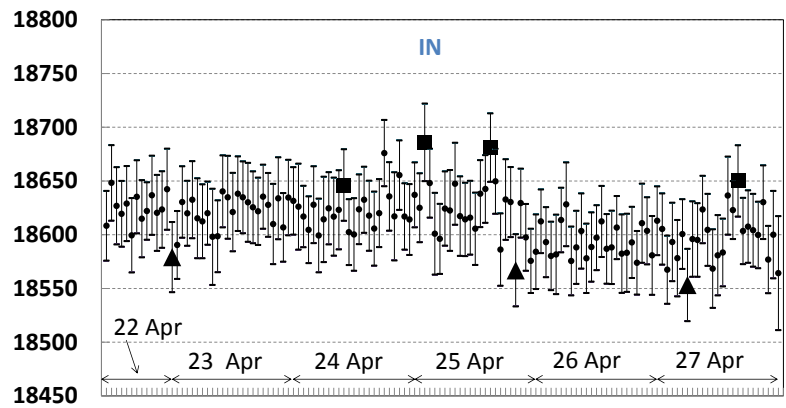

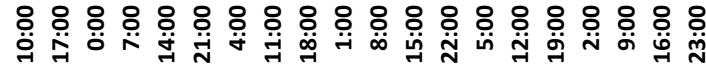
Hour

(d)

Fig. 4 Measurements along different months. a) Alternating measurements inside and outside the MFC with GM1and Ra-226 from 2015 January 7 to January 9. A first increase in cpm and a peak on January 7 at 19:00, followed by a slight oscillation, appear after transferring the source inside the cage. An abrupt drop in cpm took place after extracting the source (on January 8 at 16:00), and measurements were stable outside the shield and equal to the first means in the Figure. b) Test with Ra226 inside the shield, from 2015 February 27 to 2015 March 2. There is a similar increase of about $0.8 \%$ as in previous experiments, and two successive peaks appeared. Values at the end of the Figure are about the same as on the onset of the experiments in 2014 June. c) and d) More measurements with Ra-226 inside the MFC. c) On 2015 April 1 and April 2 fluctuations are small (differences of only $2 \sigma_{\mathrm{m}}$ ) but decay rates are up to $5 \%$ higher than in 2014 June and July. d) Fluctuations up to $6 \sigma_{\mathrm{m}}$ appeared from 2015 April 22 to 28; seven of the most different means are marked with a triangle. Values in d) are lower and more fluctuating than in c) but in both cases higher than on the onset of the experiments.

However, ten days later (Figure not represented), i.e., from 2015 March 13 at 12:54 to March 14 at 02:00 (coincidentally close to the equinox), readings taken during 850 minutes rose again, until about $18500 \mathrm{cpm}$, showing an upward trend: on this occasion cpm were higher than in Fig. 2a, Fig. 3 and Fig. $4 \mathrm{a}$ and $4 \mathrm{~b}$, thus showing that cpm increased with time, although not uniformly, to about $3.5 \%$ from the initial value on 2014 June (i.e., reaching the highest decay rates since the onset of the experiments). This trend was confirmed in the next measurements (next subsection e), with even a higher cpm.

e) More Ra-226 measurements on 2015 April

Since it seems that there exists some seasonality in the presented measurements (which has some resemblances to the observations of the authors mentioned in the Introduction section), and since an upward trend was apparent, more tests inside the cage were necessary to perform. So, in Fig. 4c one can see the measurements that were taken from 2015 April 1 to April 2 during 23 hours. In comparison with the values on 2014 July 14, 15 and 16, current data are up to $2 \%$, (360 cpm), and up to $5 \%$ higher than the first measurement (on June 23) in that Figure. Here, one can observe the highest cpm values up until this date (more than $18720 \mathrm{cpm}$ ). On the other hand, only slight fluctuations are apparent in Fig. 4c and by means of a t-test one can verify that mean value at April 1 at 19:00 is different from the means at April 1 at 12:00, 17:00, and April 2 at 00:00 and 09:00, but only at the 95\% confidence level and $2 \sigma_{\mathrm{m}}$.

In Fig. 4d (measurements taken from 2015 April 22 to April 27), fluctuations increased with time and the maximal differences reached $6 \sigma_{\mathrm{m}}$. Some of the most separated means are marked with a square and a triangle. 


\subsubsection{Some measurements with Tl-204}

The next brief analysis refers to the comparison of decay rates of Tl-204 measured at different times, both outside and inside the MFC, which are: a) measurements taken during almost 3 hours outside the box, from 2014 June 12 at 09:00 to about 12:00 (see three first mean values in Fig. 5), and b), data taken during 27 consecutive hours inside the MFC, from 2015 July 25 at 17:00, to July 26 at 19:00. In this Figure the confidence interval for each mean is also represented. All standard deviations are equal and all data groups fit a normal distribution quite well.

This Figure (as well as all previous ones where sharp changes in cpm are shown) exemplifies how cumulative skewness (starting on 2014 July 25 at 17:00 and taking the fifteen following points) deviate significantly from the values corresponding to a normal distribution. That interval includes the first dip on July 25 at 22:00 and the ten subsequent data groups.

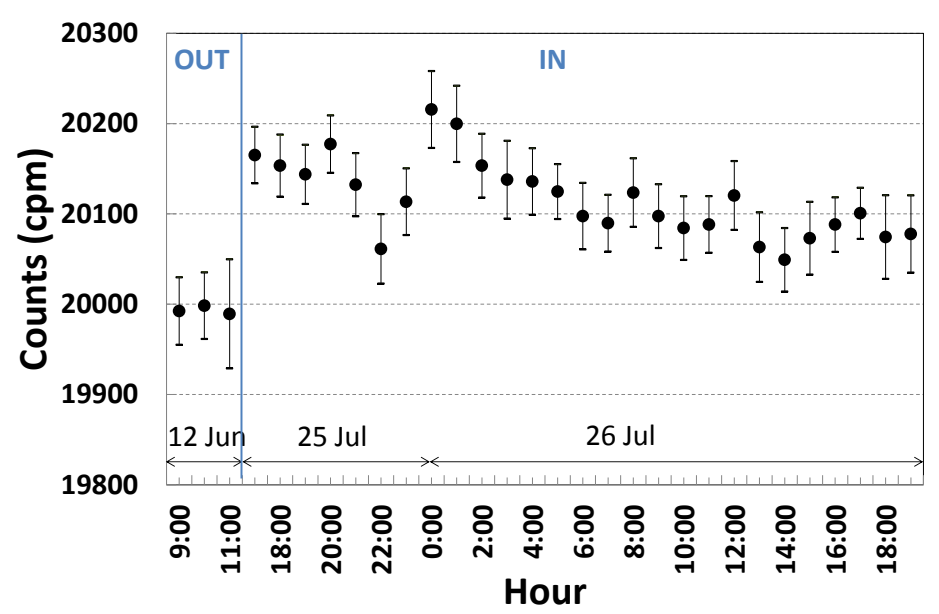

Fig.5 Tl-204 decay rates comparison both outside and inside the enclosure taken at different days and months. The first three points correspond to three hours outside the box on 2014 June 12. The following 26 measurements were taken throughout 26 consecutive hours inside the MFC, starting on 2014 July 25 at 17:00. An increase up to $1.1 \%$ can be observed after placing the GM1 inside the box. Coincidentally, a peak appears on July 26 at about 00:00, coinciding with a small peak in geomagnetic activity. Another small peak (relative to its surrounding) appears 12 hours later on July 26 at 12:00. Cumulative skewness from data before the first dip and after the first peak deviate from values representing a normal distribution.

Once more, measurement results inside the box tend to be higher than outside it: in this case, the difference is about $1.1 \%$, somewhat higher than in Fig. $2 \mathrm{~b}$. The first three tests form a homogeneous group which differs significantly from almost all the other means inside it (except for some values like July 25 at 22:00, July 26 at 13:00, and at 14:00). This can be also verified with the t-test. The difference between the initial values and the peak is greater than $8 \sigma_{\mathrm{m}}$.

The fluctuations around the peak on July 26 at 00:00 are noteworthy: a sharp decrease starts on July 25 at 20:00, reaching a minimum after two hours at 22:00; then, a sharp increase starts (also of two hours duration) with a peak on July 26 at 00:00. This also coincided with a peak in geomagnetic activity observed at that time [45]. After this peak, means from July 26 at 00:00 to 07:00 follow an exponential attenuation given by $\mathrm{y}=20223.6 \exp (-0.0009 \mathrm{x})$, and $\mathrm{R}^{2}=0.94$.

Also, as in previous cases, a Box-and-Whiskers plot (not represented) shows that outliers of the successive (one hour) data groups tend to increase with time during measurements inside the box. 


\subsubsection{Some measurements with $\mathrm{Sr}-90 / \mathrm{Y}-90$}

The third sample used to analyze decay rates was a Sr-90/Y-90 preparation. Data inside the MFC were taken on 2014 December, 2015 February, and 2015 May. But first, in order to check the stability of the measurements, data were taken during 28 hours outside the box (Fig. 6a, starting on 2015 December 9 at 11:25) and, as expected, neither statistically significant difference amongst the means nor significant difference amongst the standard deviations was detected. The grand mean was $402.6 \mathrm{cpm}$ and the standard deviation $20.1 \mathrm{cpm}$. Skewness and kurtosis were slightly out of range only in four cases.

The first 28 measurements outside the MFC were followed by two different periods on the inside (Fig. 6b): firstly during about 17 hours (starting on 2014 December 11 at 10:00) and later during 14 more hours (starting on 2014 December 15 at 9:00). The radiation was attenuated by the same bulk of the source because it was placed upside down on the tray of the lead container.

Again, after about five hours on the inside, the values started to fluctuate around the mean value. The resulting oscillations led to significant differences in mean values up to $3.1 \%$ (or $4 \sigma_{\mathrm{m}}$ ). This result differs from the other outcomes observed in previous experiments (as in Fig. 3a, and Fig. 4), where first an initial increase in decay rates took place, which was followed by subsequent oscillations. The last period of about fourteen hours inside the box ,also showed abnormal fluctuations and significant variations up to $2.5 \%$ (a t-test yields a $\mathrm{P}$ value of 0.014 ).

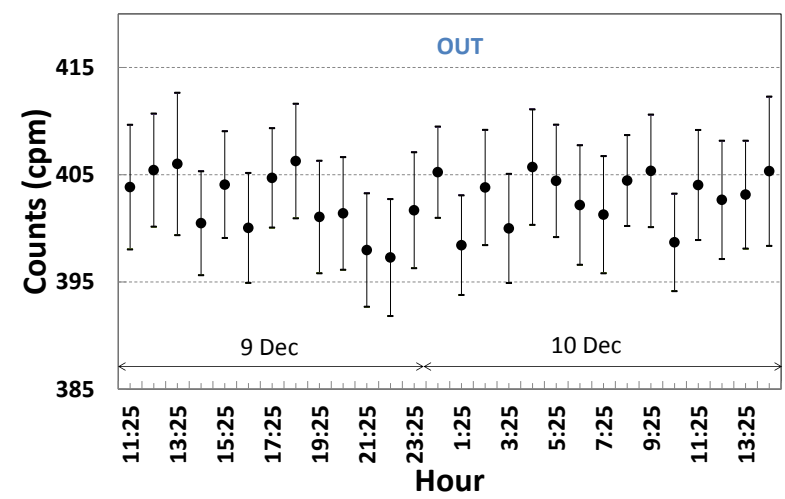

(a)

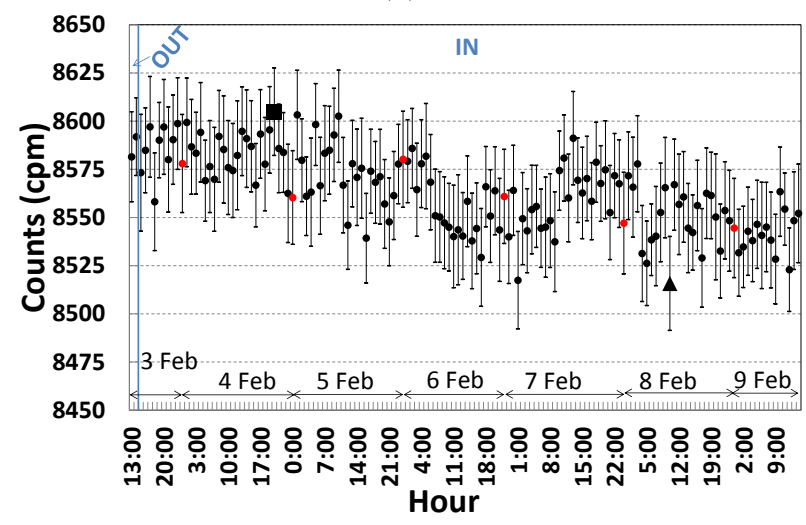

(c)

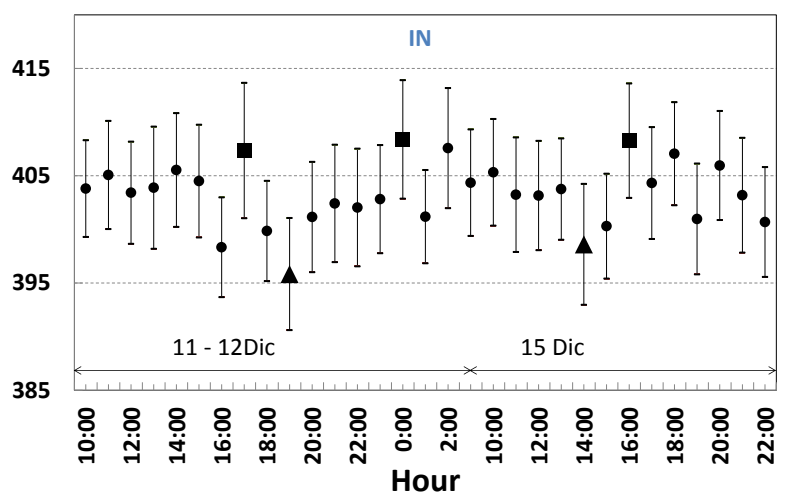

(b)

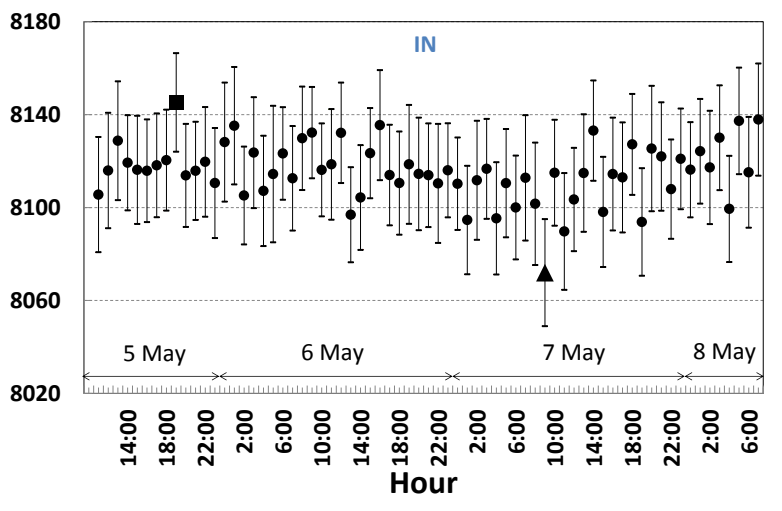

(d)

Fig. 6 Measurements outside and inside the MFC with Sr-90/Y-90. a) Preliminary measurements outside the box during 28 hours, starting on 2014 December 9. There is no significant difference amongst the means, nor there is amongst the variances. b) Measurements on 2014 December 11 and December 15 
with the sample in the inside: fluctuations appeared with differences up to $3.1 \%$ (or $>4 \sigma_{\mathrm{m}}$ ). c) Measurements inside the box from 2015 February 3 to February 9. Fluctuations appeared after 36 hours inside the MFC. At this time, maximal differences were of $1 \%$ (or $7 \sigma_{\mathrm{m}}$ ). Two of the most distant means are marked with a square and a triangle. d) Test inside the enclosure from 2015 May 5 to May 8 . Fluctuations were similar to those in 2015 February $\left(0.9 \%\right.$ or $\left.6 \sigma_{\mathrm{m}}\right)$. The average $\mathrm{cpm}$ in this test is $5 \%$ lower than in February.

Here as well, no significant differences amongst the variances were found. The values of skewness and kurtosis (for the successive data groups inside the box) are always within the limits of a normal distribution.

In Fig. $6 \mathrm{~b}$ one can observe once more that, as time passes, fluctuations appear inside the MFC in some unexpected manner. Therefore, in order to repeat the previous experiment, to try to see the previous effect again and to record decay rates during an even longer time, more measurements were taken in 2015 February and May 2015. Results are shown in Fig. 6c and Fig. 6d respectively. In these cases the recorded activity is higher because the radiation was not attenuated by the bulk of the source.

Measurements starting on 2015 of February 3 lasted for 146 hours (Fig. 6c). Similarly to previous cases, at the beginning there were no significant differences amongst the means, with almost no oscillations during the first 36 hours. However, fluctuations appeared afterwards (along with a decreasing trend), although more attenuated than in Fig. $6 \mathrm{~b}$ and with a maximum difference amongst the means of $1 \%\left(\right.$ or $\left.7 \sigma_{\mathrm{m}}\right)$.

Later on, measurements starting on 2015 May 5 (at about 11:00) lasted for almost three days (Fig. $6 \mathrm{~d}$ ). Here, fluctuations were similar to those in Fig. $6 \mathrm{c}$ and differences up to $0.9 \%$ (or $6 \sigma_{\mathrm{m}}$ ) appeared again. But, it seems important emphasize that with regard to the measured activity, a very significant drop in cpm was noticed (contrary to what happened with Ra-226 measurements): whereas in 2015 February (Fig. 6c) the grand mean was around $8560 \mathrm{cpm}$, in 2015 May (Fig. 6d) it was $8116 \mathrm{cpm}$, or 5\% lower. In the case of Ra-226, however, an increase of about 4.5\% in cpm took place from 2015 February to April (Fig. 4).

Thus, all this raises again the question on the possible seasonal effect and on the mechanism that originates such changes, which on the other hand seems to act differently depending on the type of nuclide. Therefore, since the number of results is too low to draw relevant conclusions, it is necessary to repeat the measurements as many times as possible in other months (and simultaneously with different nuclides) to check whether there are actually periods in which: a) the highest fluctuations in short periods of time take place and b), the highest (absolute) increment in activity can be observed.

Due to the different responses of Sr-90/Y-90 with regard to Ra-226 and Tl-204, another question that arises is whether it might be related to the different kind of radiation emitted by the nuclide and to the detection mechanism. While the former element only emits beta minus particles (which only interact with the gas) and do not emit gamma rays, Ra-226 emit alpha, beta and gamma rays (although alpha particles are filtered by the source envelop), and Tl-204 emits beta minus particles and low intensity X-rays.

Gamma and $\mathrm{X}$ rays of low energy interact with the gas atoms by photo-ionization, but for higher energies, the ionization of the gas is caused by electrons ejected by photo-emission at the inner surface of the cathode, which in turn causes the Townsend avalanche. One could only speculate why different types 
of radiation cause the observed anomalies (assuming that a seasonal effect is not superimposed to the other ones).

\subsubsection{Remarks about decay measurements}

Regarding the anomalous variations observed in previous experiments, it can be emphasized that they do not always start after placing the counters inside the box and at the same speed. For example, the first transient in Fig. 4a starts after two hours and only reaches a maximum two hours later (in contrast to what happens in Fig. 3a and Fig. 3b, where the upward trend starts later). Aside from this, it is also apparent that in these experiments, abnormal results can be observed not only after a time of the source having been placed inside the enclosure (a kind of time-lag), but also when the period of observation increases (see last jump in Fig. 3a).

As already pointed out, the observed changes in mean values and in strong fluctuations of activity could be explained by changes (fluctuations) in detector's dead time and recovery time. But then, the related changes in pulses' shape should be attributed to changes in the detector's time constant RC, where $\mathrm{R}$ is the resistor of the counting circuit and $\mathrm{C}$ is the counting circuit equivalent capacitance (the sum of tube capacitance along with the cables and stray capacitances). So, a hypothetical drop in RC could cause two competing effects: on the one hand, it could reduce the pulses' length and thus the dead time (favoring the increase of counting rates), and on the other hand, it could reduce the pulses' amplitudes, thus reducing the probability of pulses detection). In any case, one question is whether the counting circuit time constant experiences some changes inside the MFC and why, and also if those hypothetical changes might depend on the materials used for the electronic circuitry. A first attempt to look for such variations is performed in the next sections using a class-I multilayer ceramic capacitor.

Besides, one could also ask about the parallelism between the presented effects and the ones described in the Introduction section, and if some of the existing models (see [9]) could explain the described observations, which on the other hand, seem to happen only sometimes under unknown environmental conditions. In this regard, the possibility of a seasonal effect makes necessary to repeat these experiments in a similar way as performed by the authors mentioned in the Introduction section, i.e., along the year(s) and in coincidence with major cosmophysical events. By this means it might be checked if the MFC acts as a kind of amplifier of those effects. For now, the current and next experiments are intended to be the firsts ones of a longer series that could lead to conclusions about the variability (and periodicity) of decay rates measurements inside a MFC (either due to some effect on the measuring system, on the preparations themselves or on both).

\subsection{Spectrum of Cs-137}

The spectrum of Cs-137 was also checked both inside and outside the box. Since this was a test of preliminary character, only a few measurements were performed (in May 2000). The result is presented in Fig. 7: 


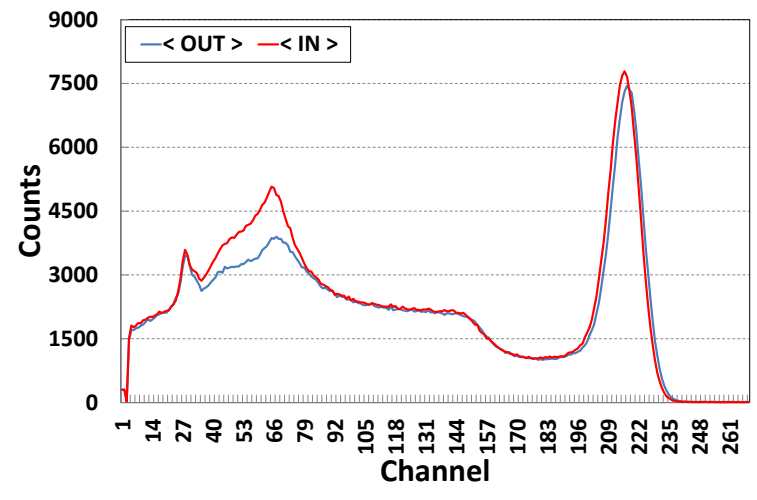

(a)

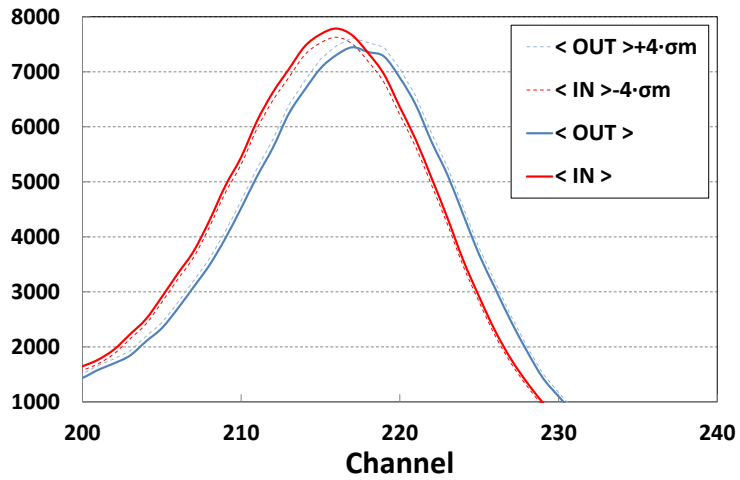

(b)

Fig. 7 Cs-137 gamma spectra comparison. a) Averaged gamma spectrum of Cs-137 both outside (blue) and inside (red) the MFC. b) Spectra photopeaks. The red (blue) dashed line is the averaged spectrum minus (plus) $4 \sigma_{\mathrm{m}}$. The photopeak region is shifted to lower energies and the difference between photopeaks is higher than $8 \sigma_{\mathrm{m}}$. Brackets are used to represent average values.

Fig. 7a presents the whole spectra obtained both outside and inside the box, whereas Fig. $7 \mathrm{~b}$ only corresponds to the photopeak region. This region, when measured inside the MFC, is shifted towards the lower energies: the photopeak that corresponds to the photon of $0.662 \mathrm{MeV}$ is displaced in at least two channels and increased in more than $8 \sigma_{\mathrm{m}}$, where $\sigma_{\mathrm{m}}$ is the standard deviation of the mean. Thus, it is apparent that the instrument reacted as if it was out of calibration both in energy (photopeak channel displacement) and efficiency (registered counts increase), which in turn could have been the response to some perturbation on the circuitry of the detector (resistors, capacitors and preamplifier). However, the valley, the Compton edge and the Compton continuum remain unaltered; the backscatter peak increases markedly due to the proximity of the MFC screen to the source and to the photomultiplier tube.

Regarding the channels displacement, it cannot be said with certainty that it was an effect of the enclosure, because data about ambient temperature variations at the time of measurements are not available. Although those values were within the comfortability limits of the laboratory and quite stable (see later, Fig. 10), one cannot ascertain whether an increase or decrease of about $2{ }^{\circ} \mathrm{C}$ could have taken place. However, such assumed variation would have not been reason enough to the increase in counts registered in the photopeak. This could be explained by an increase in after pulses, but in that case, one could ask why the possible factors that cause the after pulses [35] would be enhanced inside the enclosure.

Another observation was an increase of resolution $R_{p}$, given by [46]:

$$
R_{p}=F W H M / E_{o}
$$

where FWHM is the peak full width at half maximum and $\mathrm{E}_{\mathrm{o}}$ is the energy detected by the channel corresponding to the photopeak. Thus, it can be calculated that resolutions both outside and inside the MFC are respectively: $\mathrm{R}_{\mathrm{o}}=7.3 \%$ and $\mathrm{R}_{\mathrm{i}}=7.7 \%$. This increment in $R_{p}$ represents an increase in electronic noise generated by the circuitry inside the shield, which in turn is also related to the thermionic emissions from the phototube dynodes.

This is a complex process with many factors determining the shape of the spectrum. But as with the GM counter, one of the elements that configure the outcomes is the time constant RC of measuring 
circuit: assuming that the crystal time constant remains unchanged, the lower the photomultiplier time constant, the narrower the pulse will be, and thus, the more particles can be registered by the detector. Thus, the question rises again on whether such a circuit could be affected inside the MFC. This is analyzed in what follows.

\subsection{Low-pass filter time constant}

An input signal of $0.2 \mathrm{~ms}$ and $5 \mathrm{Vac}$ was applied to the low-pass RC filter. The USB oscilloscope was set to perform one measurement of one second duration and the input signal was scanned at a rate of 400 $\mathrm{kHz}$. So a set of 5000 measurements of both the maximum output and input voltages could be taken each second and the average was calculated. This procedure was repeated four times both outside and inside the shield, and the corresponding average values were calculated. It has to be noted that the small USB measuring device was placed also inside the MFC due to its small dimensions.

Using Eq. A.1, we obtained the average time constant $\tau=\mathrm{RC}$ values in three cases:

a) When the RC filter was transferred to the inside of the MFC, $\tau$ increased in average about $5.7 \%$.

b) When the RC setup was kept in the same place but the resistor $\mathrm{R}_{1}$ was substituted by two equal resistors $\mathrm{R}$ in series, $\tau$ increased by $5 \%$.

c) When the RC setup was kept in the same place but the resistor R was substituted by two equal resistors $\mathrm{R}$ in parallel, $\tau$ increased by $8 \%$.

Since a possible influencing factor could be the fringe capacitance between the wires of the setup and the metallic plates of the MFC, it is necessary to estimate that effect. The fringe capacitance between the wires of the setup and the metallic plate can be approximated by [47]:

$$
c_{\text {wire }} \cong c_{p p}+c_{\text {fringe }}=\frac{w \varepsilon_{d i}}{t_{d i}}+\frac{2 \pi \varepsilon_{d i}}{\log \left(t_{d i} / H\right)}
$$

where $\mathrm{w}$ is an approximation for the width of the parallel-plate capacitor; $t_{d i}$ and $\varepsilon_{d i}$ represent the thickness of the dielectric layer (distance from the wires to the MFC metallic wall) and its permittivity (air), respectively. $\mathrm{H}$ is the wire diameter. $\varepsilon_{d i}$ is the product of two terms, $\varepsilon_{d i}=\varepsilon_{r} \cdot \varepsilon_{o}$ where $\varepsilon_{r}=1$ (air relative permittivity) and $\varepsilon_{o}=8.854 \cdot 10^{-12} \mathrm{~F} / \mathrm{m}$. Approximately, the distance of the wire to the plate can be taken as $10 \mathrm{~cm}$, and the capacitor and resistor wire diameter as $0.5 \mathrm{~mm}$. With these values, the first term can be neglected and the fringe capacitance is about $12 \mathrm{pF} / \mathrm{cm}$; for a total wire length of less than $10 \mathrm{~cm}$, the parallel capacitance can be estimated as $0.1 \mathrm{nF}$, which is much smaller than the $10 \mathrm{nF}$ capacitance. Therefore, the contribution of the wire capacitance can also be neglected.

These measurements show that a circuit time constant RC can somehow be affected inside the MFC. Therefore, the next measurements were about the capacitance of the used class-I capacitor.

\subsection{Capacitance measurements}

Capacitance was measured directly with an Agilent LCR meter [48]. The used materials and procedures are described in the Appendix. Capacitor parameters were measured repeatedly both outside 
and inside the MFC, at the frequencies, $\mathrm{f}$, of $10 \mathrm{kHz}$ (medium impedance), $100 \mathrm{~Hz}$ (high impedance), and also alternating between both frequencies, always with a test signal of 1 Vac. Some complementary measurements at $\mathrm{f}=100 \mathrm{kHz}$ were also carried out. From all the parameters which are possible to measure with the LCR meter, capacitance values are the only presented.

\subsubsection{Alternating measurements outside the box at $100 \mathrm{~Hz} / 10 \mathrm{kHz}$}

In order to facilitate the description of the observations that follows, it is firstly convenient to present the response of the used capacitor when the frequency changes from $100 \mathrm{~Hz}$ to $10 \mathrm{kHz}$ and vice versa under normal working conditions (outside the enclosure). This can be seen in Fig. 8: the first part is the capacitance measured at $100 \mathrm{~Hz}($ mean $(\mathrm{m})=10.1936 \mathrm{nF}, \sigma=0.001654 \mathrm{nF}$ ) followed by the values at 10 $\mathrm{kHz}(\mathrm{m}=10.0766 \mathrm{nF}, \sigma=0.000123 \mathrm{nF})$; afterwards at $100 \mathrm{~Hz}$ and again at $10 \mathrm{kHz}$ with similar means and dispersion $\sigma$. Each point (in this Figure and in the following) represents the mean of 180 measurements taken each hour and the confidence interval (given by $\pm 2 \sigma_{\mathrm{m}}$ ) is represented. Instrument's accuracy at 100 $\mathrm{Hz}$ is $0.3 \%$ of the reading and $0.08 \%$ at $10 \mathrm{kHz}$ and $100 \mathrm{kHz}$.

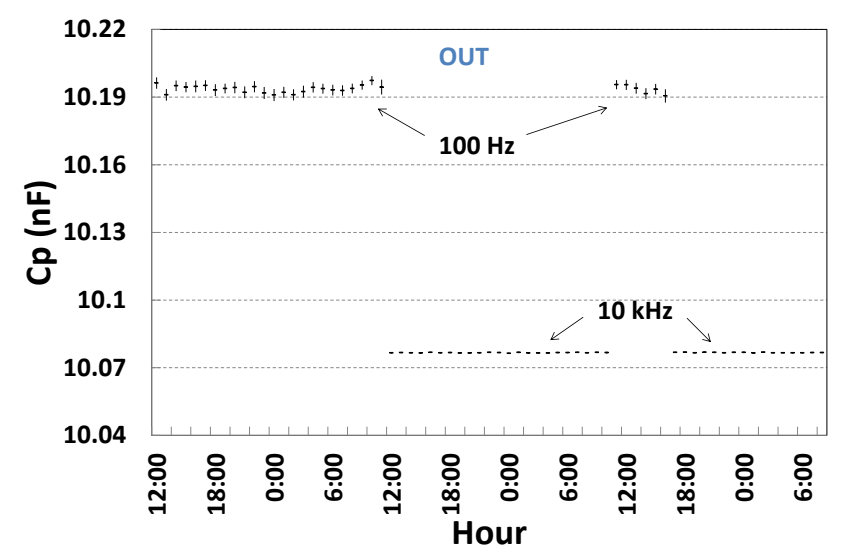

Fig. 8 Alternating measurements outside the shield at $100 \mathrm{~Hz}$ (high values about $10.19 \mathrm{nF}$ ) and $10 \mathrm{kHz}$ (lower values about $10.07 \mathrm{nF}$ ) on 2015 April. When changing the frequency, capacitance changes abruptly, with no time-lag effect.

So, the capacitance changes sharply when the frequency changes from $100 \mathrm{~Hz}$ to $10 \mathrm{kHz}$ and viceversa (the same happens when one of these frequencies is $100 \mathrm{kHz}$ ). At these frequencies, the dielectric of this capacitor does not show any time-lag effect caused by the frequency variations. However, it will be shown (section 2.5.3.1) that this is not always the case, particularly after having undertaken capacitance measurements at $10 \mathrm{kHz}$ inside the MFC.

Once seen how this capacitor behaves under these frequency variations (which includes the implicit self-heating effect), measurements were taken at $10 \mathrm{kHz}$ but alternating the location from the outside to the inside of the MFC and vice versa (after that, the same was repeated at $100 \mathrm{~Hz}$ and $100 \mathrm{kHz}$ ).

\subsubsection{Alternating measurements inside and outside the box at $10 \mathrm{kHz}$}

Capacitance was measured at $10 \mathrm{kHz}$ inside and outside the MFC, a total of five times successively (Table 1) on 2015 February and March. In each one of all the tests performed inside the MFC (i.e., tests 
$1_{10 \mathrm{k}}, 3_{10 \mathrm{k}}, 5_{10 \mathrm{k}}$ ), the means (or medians) and variances were equal. However, one or more of those statistics showed significant oscillations when the capacitor was transferred to the outside (but only in some of the successive data groups).

In Table 1 it can be seen that capacitor worked at the frequency $\mathrm{f}=10 \mathrm{kHz}$ during 346 hours, of which 235 were spent inside the MFC, i.e., under conditions that have altered the measurements of decay rates and the spectrum of a preparation.

Table 1 Successive capacitance measurements at $\mathrm{f}=10 \mathrm{kHz}$ both inside and outside the cage.

\begin{tabular}{cccccc}
\hline Test $_{\mathrm{f}(\mathrm{Hz})}$ & Location & Dates & $\begin{array}{c}\text { Time } \\
\text { (hours) }\end{array}$ & $\begin{array}{c}\text { Mean } \\
(\mathrm{nF})\end{array}$ & $\begin{array}{c}\text { Std. } \\
\text { deviation }\end{array}$ \\
\hline $1_{10 \mathrm{k}}$ & IN & 12-16 Feb. & 92 & 10.0752 & 0.002192 \\
$2_{10 \mathrm{k}}$ & OUT & 20-23 Feb. & 65 & 10.0757 & 0.002250 \\
$3_{10 \mathrm{k}}$ & IN & 27 Feb.- 2 Mar. & 66 & 10.0751 & 0.002179 \\
$4_{10 \mathrm{k}}$ & OUT & 4-6 Mar. & 46 & 10.077 & 0.002148 \\
$5_{10 \mathrm{k}}$ & IN & 6-9 Mar. & 77 & 10.0768 & 0.080336 \\
\hline
\end{tabular}

On the other hand, when comparing the five groups (of Table 1) to each other, the result is that all the five groups fit a normal distribution but the variances in tests inside the box are different from those on the outside. Besides, medians (and means) fluctuate as shown in Fig. 9a: here also significant changes take place when the source changes from inside to outside the MFC and vice versa. The central line in Fig. 9bis the moving average of $\mathrm{Cp}$ values, and one can see the jump when the capacitor changes from inside the box (test $3_{10 \mathrm{k}}$ ) to the outside (test $4_{10 \mathrm{k}}$ ).

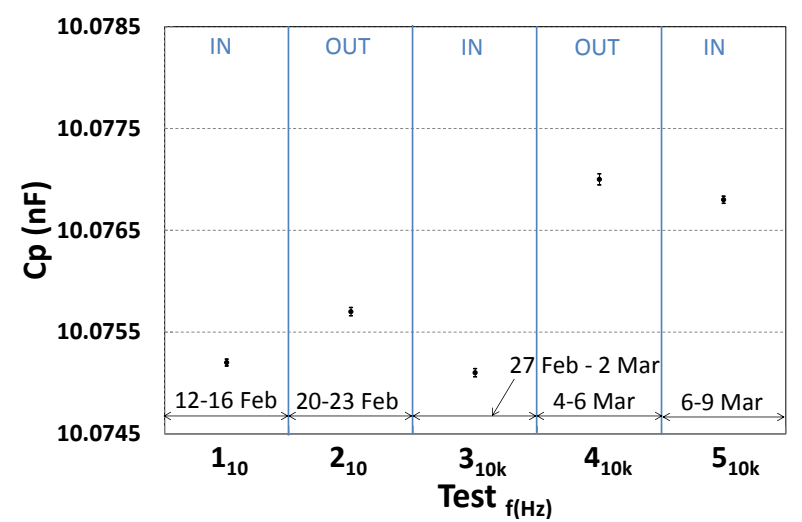

(a)

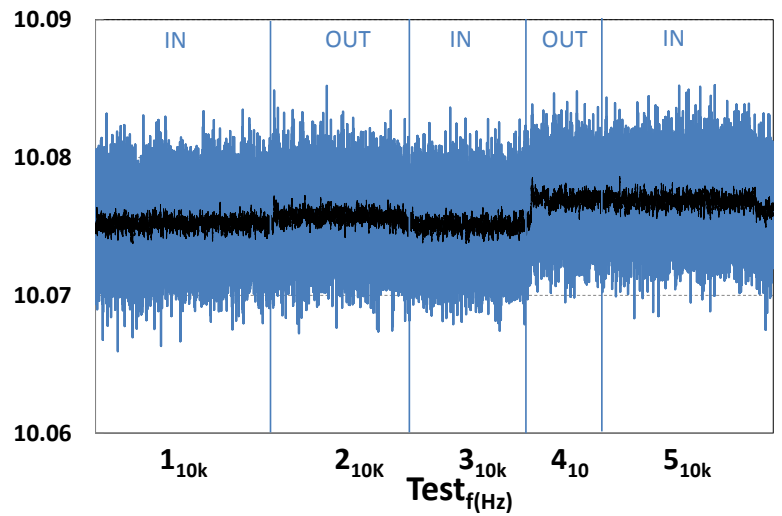

(b)

Fig. 9 Capacitance measurements on 2015 February and March. Five alternating tests inside and outside the box at $10 \mathrm{kHz}$. a) Mean values of $\mathrm{Cp}$, which fluctuate similarly to the medians. Although they are within instrument's accuracy, there are statistically significant differences between the medians. When the capacitor changes its location from the inside to the outside, there is always an increase in Cp but of different intensity. The capacitance drops again when it is transferred to the inside. b) Moving average of $\mathrm{Cp}$ (central line); the jump from test $3_{10 \mathrm{k}}$ to test $4_{10 \mathrm{k}}$ is apparent.

$\mathrm{Cp}$ values in Fig. 9a are within accuracy limits, but as in the case of decay rates measurements, differences and fluctuations in results always appear after having been inside the MFC for a time. These 
results raise the question on a possible slight change in dielectric state of polarizability. This seems to be also the case in the next section.

\subsubsection{Alternating measurements both outside and inside the box at $100 \mathrm{~Hz}$}

Subsequently, in a similar way to what was done at $10 \mathrm{kHz}$, more measurements of capacitance were performed at the frequency of $100 \mathrm{~Hz}$ during the months of 2015 March and April. A summary of these next tests and its duration is given in Table 2 .

Table 2 Successive capacitance measurements both outside and inside the box at $\mathrm{f}=100 \mathrm{~Hz}$.

\begin{tabular}{cccccc}
\hline Test $_{\mathrm{f}(\mathrm{Hz})}$ & Location & Dates & $\begin{array}{c}\text { Time } \\
\text { (hours) }\end{array}$ & $\begin{array}{c}\text { Mean } \\
(\mathrm{nF})\end{array}$ & $\begin{array}{c}\text { Std. } \\
\text { Deviation }\end{array}$ \\
\hline $1_{100}$ & OUT & 11-12 Mar & 19 & - & - \\
$2_{100}$ & IN & 12-20 Mar & 193 & 10.1892 & 0.017276 \\
$3_{100}$ & OUT & 20-27 Mar & 173 & 10.1877 & 0.015328 \\
$4_{100}$ & IN & 27 Mar - 07 Apr & 264 & 10.1858 & 0.015505 \\
$5_{100}$ & OUT & 07-14 Apr & 168 & 10.1917 & 0.016089 \\
\hline
\end{tabular}

These tests are described in what follows.

\subsubsection{First measurements outside and inside the box at $100 \mathrm{~Hz}$}

In tests $1_{100}$ and $2_{100}$ (Table 2), two respective transients which showed significant changes surpassing instrument's accuracy took place. With regard to test $1_{100}$ (11-12 Mar), it might be important to notice that the capacitor had been working before inside the box at $10 \mathrm{kHz}$ (tests $1_{10 \mathrm{k}}, 3_{10 \mathrm{k}}$, and $5_{10 \mathrm{k}}$ in Table 1 ), and later, the frequency was changed to $100 \mathrm{~Hz}$. The obtained response can be seen in Fig. 10 from the beginning of the curve until its maximum, where $\mathrm{Cp}$ values stabilize. The $\mathrm{Cp}$ value at the beginning is $\leq$ $10.13 \mathrm{nF}$, which is closer to the values at $10 \mathrm{kHz}$ (i.e. about $10.0760 \mathrm{nF}$, as seen in Fig. 8), and does not correspond to the $\mathrm{Cp}$ values at $100 \mathrm{~Hz}$ (Fig. 8 and Table 1). In other words, when the frequency was changed to $100 \mathrm{~Hz}, \mathrm{Cp}$ did not change abruptly to $10.2 \mathrm{nF}$, as seen in Fig. 8. Instead, it took about 19 hours to reach the most characteristic value of $10.21 \mathrm{nF}$. The increment during this transient was of about $0.07 \mathrm{nF}$ (or $0.7 \%$ ), which largely exceeds instrument's accuracy. This gradual variation in Cp values does not correspond to the change in ambient temperature (just nearby the capacitor) which is also shown in Fig. 10.

Regarding this first transient, all happens as if after working at $10 \mathrm{kHz}$ during that specific long period of time (inside and outside the MFC) the dielectric structure had become "reorganized", acquiring another (and lower and relatively anchored) state of polarization. This apparent remnant state seemed to disappear slowly with time (similarly to a dielectric relaxation response), thus returning at the higher state of polarization corresponding to the frequency of $100 \mathrm{~Hz}$ (which in turn justifies the higher capacitance).

After reaching its characteristic value at $100 \mathrm{~Hz}$ at the outside, the capacitor was placed immediately inside the box for the next 193 hours, from 2015 March 12 to March 20 (test 2100, Table 2); the corresponding $\mathrm{Cp}$ values are the ones after the peak in Fig. 10. 
Now another anomalous response was observed because its capacitance decreased almost continuously, and only after eight days (about 189 hours) the drop in Cp values exceeded the limits of instrument's accuracy $( \pm 0.0318)$ plus the error due to ambient temperature variations. This drop in $\mathrm{Cp}$ values resembles the ones observed in Fig. 9a after placing the capacitor inside the box, but now the change is more significant.

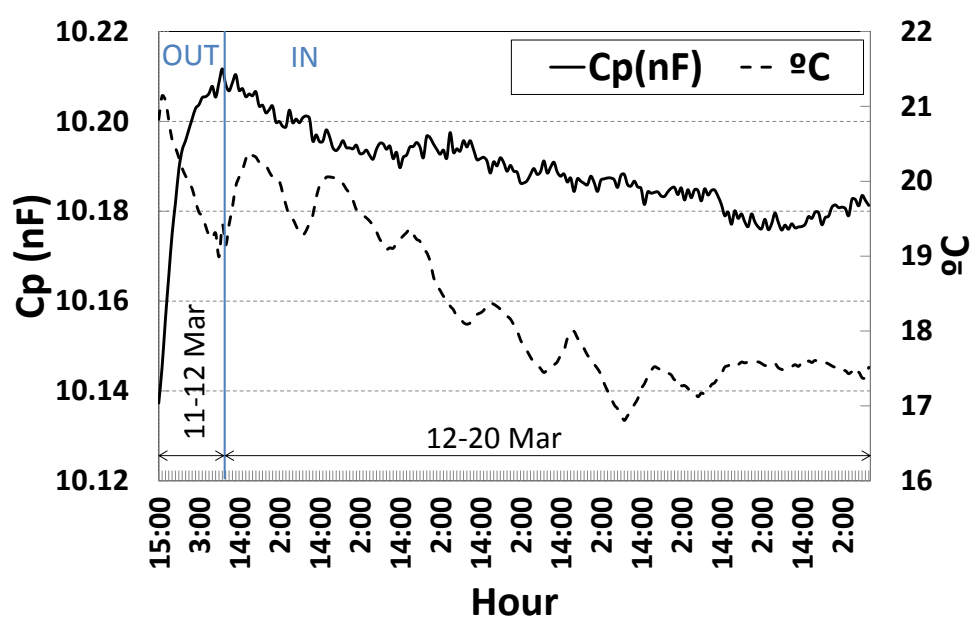

Fig. $10 \mathrm{Cp}$ (solid line) and ambient temperature (dashed line) outside and inside the box at $100 \mathrm{~Hz}$ from 2015 March 11 to 2015 March 20 (tests $1_{100}$ and $2_{100}$, Table 2). The first ascending part of the curve (test $1_{100}$, outside the box) develops during 19 hours instead of taking place instantaneously, and the increase is of about $0.07 \mathrm{nF}$. The descending part (test $2_{100}$, inside the box) takes place during the following 193 hours, and the difference between maximum and minimum values is 0.036 , which also exceeds the accuracy limit of the instrument.

Since the temperature coefficient is $\pm 30 \mathrm{ppm} /{ }^{\circ} \mathrm{C}$, the error due to temperature deviation from $25^{\circ} \mathrm{C}$ is $\pm 0.0012{ }^{\circ} \mathrm{C}$, thus, the overall error for $\mathrm{Cp}$ is $\pm 0.033 \mathrm{nF}$, which is less than the observed difference between maximum and minimum $\mathrm{Cp}$ values.

On the other hand, this behavior cannot be correlated with ambient temperature variations either, as it can be deduced form different simple regression models.

\subsubsection{Comparison of tests outside and inside the box at $100 \mathrm{~Hz}$}

In Fig. 11 the results of tests $2_{100}$ to $5_{100}$ of Table 2 are shown. These measurements were performed almost continuously, and those taken on the inside were longer because it was assumed that anomalies appeared after a period long enough inside the MFC. Fig. 11a shows all the successive means, starting with the ones of test $2_{100}$, which in turn is the descending part in Fig. 10.

An ANOVA analysis and a variance check was performed with data of tests $2_{100}$ to $5_{100}$. Since one result was that all variances were different from each other, a medians comparison test was selected (means are very close to medians) and the results are presented in Fig. 11b.

In Fig. $11 \mathrm{~b}$, one can see that the median of test $3_{100}$ (outside) remains at a lower level after the drop experienced inside the box after test $2_{100}$ (inside) although the corresponding values tend again to increase; this resembles to what happened in the first ascending part of Fig. 10. Later, the decrease 
observed in test $4_{100}$ (inside) is even more significant (and resembles to the second part of Fig. 10). But this time, for some reason, capacitance recovers in test $5_{100}$ (outside) a value similar to its initial values shown in Fig. 8.

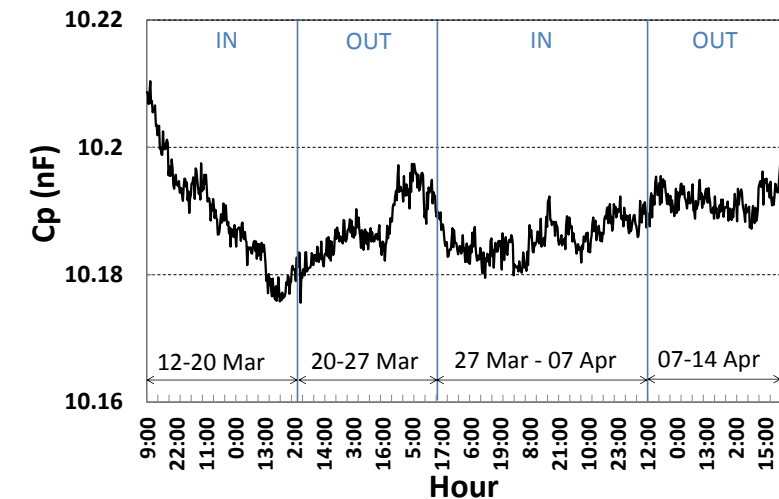

(a)

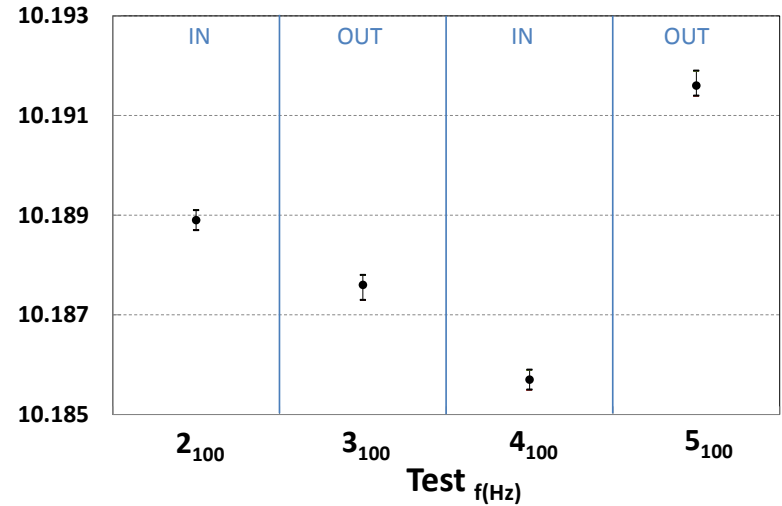

(b)

Fig. 11 Comparison of test $2_{100}$ to $5_{100}$ of Table 2. a) Means of each successive hour during tests $2_{100}$ to $5_{100}$. On the outside there is an apparent upward trend. b) Medians of tests $2_{100}$ to $5_{100}$ are different. Values inside the MFC tend to decrease.

Tests $3_{100}$ and $5_{100}$ (outside) followed immediately to tests $2_{100}$ and $4_{100}$ (inside), and there was no sudden change in $\mathrm{Cp}$ mean values, only a slow increase (which remained within the accuracy limits), approaching to the values observed in both Fig. 8 and Fig. 10, corresponding to the capacitance at 100 $\mathrm{Hz}$.

Moreover, it is noteworthy that the standard deviation in test $2_{100}$ is $0.017276 \mathrm{nF}$, which is much higher than the $\sigma$ value $0.001654 \mathrm{nF}$ observed in Fig. 8. Similarly, in test $4_{100} \sigma$ is also one order of magnitude higher than in Fig. 8.

\subsubsection{Alternating tests inside the MFC at $100 \mathrm{~Hz}$ and $100 \mathrm{kHz}$}

From previous tests, it can be suspected that anomalous dielectric responses can appear inside the MFC, which in turn seem to be related to the used frequency. But also, it seems to be related to the shield and to the dates when the measurements were taken, and thus, to some environmental uncontrolled factor. Therefore, in order to repeat (at a different time) and to complement previous tests both inside and outside of the enclosure, more Cp measurements were taken at frequencies of $100 \mathrm{~Hz}$ and $100 \mathrm{kHz}$ (Fig. 12), from 2015 June 17 to 2015 July 3.

The two new tests at $100 \mathrm{~Hz}$ (one outside and one inside the box) showed the highest and most significant differences (Fig. 12a); the corresponding measurements were taken on 2015 June 18 and on 2015 June 25 respectively. A t-test yielded that both the medians and the variances are statistically different: $10.2781 \mathrm{nF}$ for test $6_{100}$ (outside) and $10.2001 \mathrm{nF}$ for test $7_{100}$ (inside), and again, $\mathrm{C}_{\mathrm{p}}$ dropped and outliers increased. The difference of about $0.078 \mathrm{nF}$ for the medians (and means) is similar to what obtained in Fig. 10 and is significantly greater than the instrument's accuracy limit.

Besides, five alternating tests at $100 \mathrm{kHz}$ also showed significant differences between the means, although with equal variances, normal distributions and values within instruments accuracy limits (Fig. 
12b). Outliers tend to be more abundant in the inside. Similarly to tests at $10 \mathrm{kHz}$, some results are statistically different when changing from inside to outside the MFC and vice versa, but do not surpass instrument's accuracy, which until now has been only observed at $100 \mathrm{~Hz}$. It would be worth analyzing what would happen at a voltage of $5 \mathrm{Vac}$ and at a lower frequency.

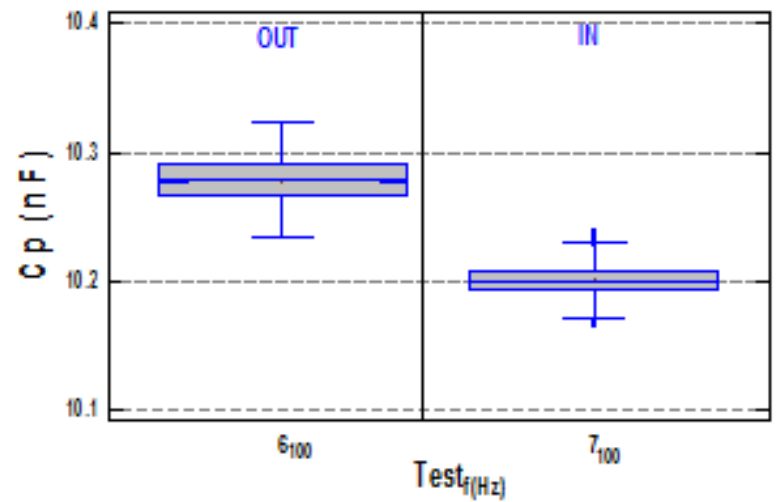

(a)

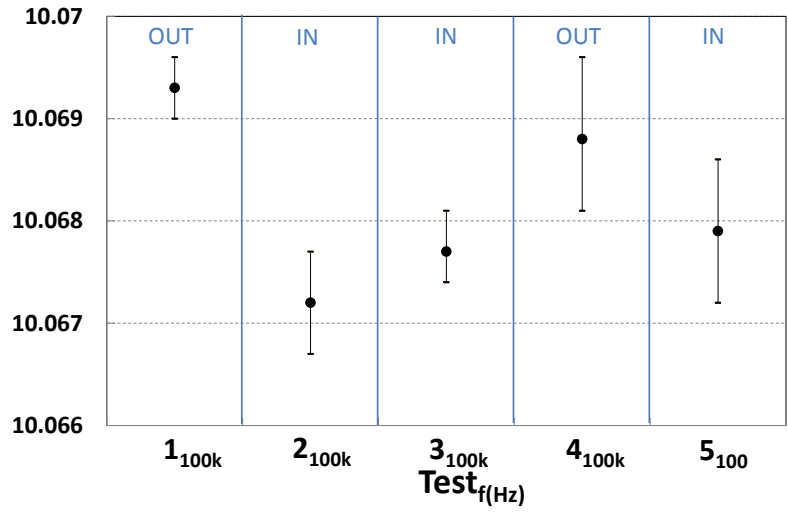

(b)

Fig. 12 Comparison of tests inside and outside the box at $100 \mathrm{~Hz}$ and $100 \mathrm{kHz}$. a) Two tests at $100 \mathrm{~Hz}$ performed on 2015 June 18 and on 2015 June 25. Cp drops inside the cage. b) Five alternating tests (inside and outside) at $100 \mathrm{kHz}$; measurements between 2015 June 18 and 2015 July 2. Capacitance decreases inside the MFC (and outliers tend to increase), but less than at $100 \mathrm{~Hz}$.

\subsubsection{Cable capacitance measurements}

The connecting cables between the GM counter tube and the scaler have a capacitance which contributes to the pulses' shape and amplitude. Therefore, the cable capacitance (with another type of dielectric), between the GM1 and the scaler, was measured both outside and inside the box at a frequency of $100 \mathrm{~Hz}$. Results are presented in Fig. 13. The first hour corresponds to measurements outside the enclosure whereas the following five hours elapsed on the inside (Fig. 13a). An ANOVA analysis shows that all the variances were different but the medians weren't.
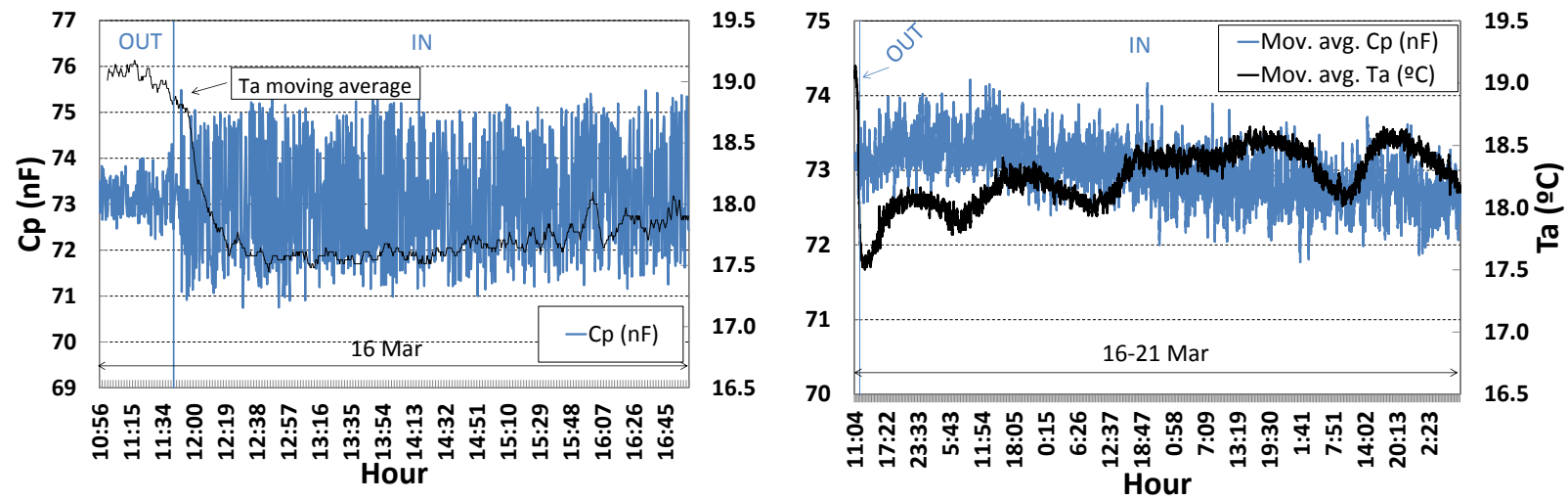

Fig. 13 Cable capacitance (Cp) outside and inside the shield. Moving average of ambient temperature $\mathrm{Ta}$ is also shown. a) First hour outside the enclosure and the following five hours inside it. The increase of noise inside the MFC is apparent, but the median is the same for all the six data groups. b) Cp and Ta moving averages along almost five days. After a slight increase at the beginning of the measurements, Cp decreases along the days. 
Fig. 13b shows how $\mathrm{Cp}$ varies along the days: after a slight but significant increase during the first day, Cp diminishes. So, the median on 2016 March 16 was 73.14 pF, whereas five days later it was 72.44 $\mathrm{pF}$ and the standard deviations 1.3331 and $1.778 \mathrm{pF}$ respectively, with no correlation with ambient temperature. This was a statistically significant decrease of about $0.7 \mathrm{nF}$ (or 1\%), greater than the decrease observed in capacitor's capacitance at $100 \mathrm{~Hz}$ (i.e. $0.078 \mathrm{nF}$ or $0.7 \%$ ). But although the cable capacitance diminishes, it is only a small portion of the cable (about $20 \mathrm{~cm}$ ) which is placed inside the box; therefore, the overall cable capacitance reduction can be estimated in only $0.2 \mathrm{pF}$. This is a small variation compared with the total stray capacitance (cable plus tube capacitances, i.e., > $(73+3.5) \mathrm{pf})$ ).

The referred capacitance changes cannot explain the observed changes in decay rates measurements. This can be explained as follows. On the one hand, the counter tube dead time $\left(t_{d}=230 \mu \mathrm{s}\right)$ implies losses up to 7.4\% in counts (as in the GM counts shown in section 2.2.1., for a typical count rate of 300 cps), which can be computed from:

$$
r_{c}=\frac{r}{1-r t_{d}}
$$

where $r$ is the measured counting rate and $r_{c}$ is the rate that could be measured without losses. Conversely, a reduction in losses of $3 \%$ would require a reduction of dead time and hence a reduction of pulses length (duration) of about $97 \mu \mathrm{s}$ which is not realistic. An estimation of the pulse length reduction (and pulse amplitude increase) due to a capacitance reduction can be obtained from a simulation of the pulse shape by means of the counter equivalent circuit [49]. With this method one can calculate that a capacitance reduction of $1 \mathrm{nF}$ only represents a pulse length reduction of about $1.5 \%$ and a pulse amplitude increase of $0.5 \%$.

An analysis of how a MCA pulse shape $\mathrm{P}(\mathrm{t})$ changes due to the detector capacitance variations can be performed by means of the pulse equation $[35,46]$ :

$$
P(t)=\frac{Q}{C} \frac{\tau}{\tau-\tau_{c}}\left[\exp \left(\frac{t}{\tau}\right)-\exp \left(\frac{t}{\tau_{c}}\right)\right]
$$

Where $\mathrm{Q}$ is the charge of one impulse, $\mathrm{C}$ is detector's capacitance; $\tau$ and $\tau_{c}$ are the detector and crystal time constants respectively. From (5) it can be seen that small changes in capacitance like the ones mentioned above do not change the pulse height and length significantly.

\subsection{Remarks about previous capacitance and decay measurements}

In section about decay measurements, it was asked if detectors circuitry could actually be altered inside the MFC. It has been shown that capacitance (of a class-I multilayer passive component) can experience slight but significant changes inside the enclosure which cannot be attributed to the ambient temperature variations existing in the laboratory. Aside from this, if those changes could be explained by a variation in dielectric's polarizability, it should be explained how this can happen, and also which is the mechanism that causes the observed variations. If we could actually discard trivial reasons, one should analyze dielectric's polarizability equations which characterize those materials [50]. And if this could be a reason, another question would arise on the possible response of other types of dielectrics: in that case, 
one could expect different capacitance changes depending on the used dielectric. In this regard, preliminary measurements have been shown in previous paragraph, where significant changes in cable capacitance (with a different type of dielectric) have been measured, but this should be tested in more experiments with more types of capacitors (including the GM counter tube).

Regarding the experiments with detectors, it has been shown that environmental parameters cannot explain the observed changes. But since the capacitor and cable capacitance can experience significant changes inside a MFC, this could explain a portion of the observed variations in count rates, because the time constant of measuring circuits can experience slight but significant changes. This affects pulses' shapes and hence, the number of recorded counts but only in a small portion which does not explain the total observed increase in cpm.

Moreover, if the observed capacitance changes were related to variations in electrical forces inside the materials, one could ask if other parameters like the work function in GM cathode and MCA dynodes could also be affected, which in turn might explain the appearance of after pulses.

\section{Summary and conclusions}

In this paper we have shown anomalous results when taking measurements of background radiation, radioactive decay rates, the gamma spectrum of a source, the time constant of a low-pass RC filter and of capacitors' capacitance inside a metallic screen (in the shape of a modified Faraday cage), as compared to previous initial measurements on the outside.

The observed changes were statistically significant variations of the initial mean (or median) values taken outside the MFC. Other statistics like variance, skewness and kurtosis changed significantly, as well as the presence of outliers which tend to increase inside the MFC.

Regarding radiation measurements (taken with a Geiger-Müller counter tube), results were different depending on whether the measurements were the background counts, or decay rates of Ra-226, T1-204 or Sr-90/Y-90. Specifically, background counts showed changes from $12 \mathrm{cpm}$ to $18 \mathrm{cpm}$.

Decay rates usually increased or oscillated (up to 3\%) after placing the GM counter tube inside the box. However, the different nuclides yielded different responses as if it were dependent on the type of interaction between the radiation to be measured (electromagnetic radiation or particle) and the counter tube. Besides, rates did not changed immediately and with the same speed; they usually only did after several hours of having been placed inside the MFC; sometimes peaks in activity occurred coincidentally around 00:00 hours. These changes in decay rates varied along the months up to 5\%, and were different both in intensity and sign depending on the used nuclide: Ra-226 increased whereas Tl-204 decreased. It was checked that rates were not significantly dependent on environmental factors like temperature, pressure, air density or high voltage instability.

The spectrum of a nuclide like Cs-137 (taken with a MCA) was also altered inside the MFC: the photopeak increased in more than $8 \sigma_{\text {avg }}$ (or by about $4.5 \%$ ), as if the instrument had gone out of calibration. Thus, it was apparent the increase of noise inside the instrument when placed inside the box, probably in form of after pulses, which might have also caused an increase of its resolution. 
Regarding the RC filter time constant using a class-I capacitor, increments of more than 5\% were measured depending on the configuration of the filter, and using a voltage signal of $5 \mathrm{Vac}$.

Direct capacitance measurements using a LCR meter at $100 \mathrm{~Hz}, 10 \mathrm{kHz}, 100 \mathrm{kHz}$, and a test signal of 1 Vac showed variations when changing the capacitor location from inside to outside the MFC (or vice versa), which were statistically significant but remained within instruments accuracy limits. However, at $100 \mathrm{~Hz}$, the highest and statistically significant variations up to $0.7 \%$ were observed, which also surpassed instruments accuracy limits. Cables capacitance also showed a slight but significant decrease with time, which contributes to a small variation in detectors' time constant. Since the capacitor and cable capacitance of the measuring devices show significant variations inside the MFC, it is plausible that these changes are a factor that influence decay rates measurements and spectrum shapes. But this change does not explain all the observed increase in cpm. Therefore, it remains to be clarified which is the factor (and its nature) which determines not only the observed changes in capacitance, but also the rest of the observed variability in cpm measurements.

Even though we statistically confirmed that the fluctuations and jumps observed in our experiments carried out in the modified Faraday cage are a systematic and measurable effect, these fluctuations cannot be explained by any of the environmental effects we have considered and measured. Further work is needed to identify whether additional effects (unknown to us at present) are responsible for these fluctuations. 


\section{Appendix: Materials and Methods}

The experimental materials, setups and procedures are described in what follows.

A.1) Radioactive samples, detectors and screens

Four radioactive sources of Ra-226, Tl-204, Sr-90/Y-90 and Cs-137 were used. The three first were used with the Geiger-Müller counters (GM), whereas the latter was used with the multichannel analyzer (MCA). The first source was a sealed Ra-226 preparation manufactured by Amersham/Searle, model $184100, \mathrm{~T}_{1 / 2}=1600 \mathrm{y}$, and of $0.9 \mu \mathrm{Ci}$ activity. This nuclide disintegrates mainly by alpha emission to the $186 \mathrm{keV}$ level and to the ground state level of Rn-226 (probabilities 5\% and 95\% respectively). The decay chain yields alpha, beta, gamma and X-rays emissions from daughter nuclide. Alpha particles appear with a kinetic energy up to $4.8 \mathrm{MeV}$ and the energies of the other emissions can be found in the literature $[51,52,53]$.

The activity of the Tl-204 source was about $1 \mu \mathrm{Ci}$. This nuclide disintegrates by beta minus emission (97.1\%) and by electron capture (EC) transition (2.9\%) to the ground states of $\mathrm{Pb}-204$ and $\mathrm{Hg}-204$. The respective energy emissions are $763.7 \mathrm{keV}$ and $347.5 \mathrm{keV}$ and $\mathrm{T}_{1 / 2}=3.78 \mathrm{y}$. As a consequence of the $\mathrm{EC}$ transition X-rays are emitted with energies between 8.721 and $87.911 \mathrm{keV}[54,55]$. From this radiation, only $20 \%$ of the photons have energies lower than $25 \mathrm{keV}$ (about $8-15 \mathrm{keV}$ ), and therefore there is a significant chance to interact with the gas, thus initiating ionization of the gas in the Geiger-Müller tube and consequently the Townsend avalanche. But detection efficiency of the remaining $80 \%$ of the photons (with energies between 68 and $88 \mathrm{keV}$ ) is as low as a few per cent, thus leaving most of the radiation passing through the tube without causing ionization. However, these highly penetrating rays (> $25 \mathrm{keV}$ ) can be detected when they eject electrons from the inner surface of the cathode $[35,56]$.

The third source was a Sr-90/Y-90 preparation of $0.1 \mu \mathrm{Ci}$ activity: it is practically a pure beta particle source. Sr-90 disintegrates by beta minus emission $(546.0 \mathrm{keV})$ to the ground state of $\mathrm{Y}-90$, which in turn disintegrates also by beta minus emission $(2284.0 \mathrm{keV})$ to the $\mathrm{Sr}-90$ fundamental level. Half-lives are $28.79 \mathrm{y}$ and $64.1 \mathrm{~h}$ respectively. Gamma-photon emission from the decay of Y-90 is of $1.761 \mathrm{MeV}$, but it can normally be ignored because it is very infrequent (only $0.02 \%$ ). Therefore, the main mechanism of detection of these rays is by the direct ionization of the gas atoms.

These isotopes are useful for our purposes because, as shown in the literature, they seem to be sensitive to whatever influence causes the alteration in decay rates $[9,14]: \mathrm{Ra}-226$ is one of the sources which exhibit an annual variation in decay rates, and since Tl-204 and Sr-90/Y-90 are also beta-radiation emitters, they are also appropriate because oscillations and periodicities in decay rates seem to appear primarily in this kind of nuclides [10].

Additionally, one Cs-137 preparation of $5 \mu \mathrm{Ci}$ activity was used. It was manufactured by Rotem Industries Ltd, model No.A-80080 and reference RS-214. Cs-137 disintegrates by beta particles of intermediate energy and the count rate includes conversion electrons from the $0.662 \mathrm{MeV}$ gamma ray [47]. This energy is characteristic of the Cs-137 spectrum, observable in the corresponding photopeak.

Radiation emitted by the radiation sources could be detected by two GM counter tubes and one multichannel analyzer. GM counter tubes are type ZP1431 for $\beta$ and $\gamma$ detection [57]. This type of radiation detectors are very robust and stable systems to perform decay rates measurements $[10,37,38]$. 
The fill gas is an inert gas (argon or neon, with ionization potentials of about 16 and $21.5 \mathrm{eV}$ respectively) mixed with a halogen for quenching purposes (usually chlorine or bromine), with a lower ionization potential than the inert gas $[35,38]$. Currently, cathodes are metals with high atomic numbers and work functions which are significantly lower than the gas ionization potential (chrome iron as in this type of tube) which inhibits the production of secondary electrons. The anode-cathode capacity of the tube is about $3.5 \mathrm{pF}$. On the other hand, GM maximum plateau slope is $0.04 \% / \mathrm{V}$, the plateau length is about $250 \mathrm{~V}$ and its maximum dead time is $230 \mu \mathrm{s}$.

The stability of GM counters (and other detectors), with respect to environmental factors (temperature, pressure and humidity), has been well studied $[10,58]$. But in addition, data provided by the counter tube supplier (Sharon Morris, Centronic Ltd, private communication, 2015) about activity measured at different temperatures of $23,50,60$, and 70 degrees centigrade, yields a linear regression line: $\mathrm{cpm}=100873+53.1367 \mathrm{~T}\left({ }^{\circ} \mathrm{C}\right)$, with $\mathrm{R}^{2}=0.98$. Thus, temperature variations of $\pm 5^{\circ} \mathrm{C}$ would yield $\pm 0.3 \%$ error in measured cpm: this temperature variation span is higher than the temperature variations observed during the experiments and therefore the errors due to these changes will be lower than $0.3 \%$. As it will be shown in the Results section, the observed changes in cpm are higher than this value. It should be stressed at this point that the environmental conditions in the laboratory ensured a small temperature variability of less than $\pm 5^{\circ} \mathrm{C}$; along one day it can vary about $1^{\circ} \mathrm{C}$ (see Fig. 10 ). On the other hand, ambient pressure oscillations cannot be a factor influencing the counter stability because the fill gas is insensitive to those variations [59].

One could also think that air density variations due to changes in ambient temperature and relative humidity could be a reason for the possible oscillations in decay measurements. But it has been demonstrated [10] that this is not an issue when analyzing periodic changes in $\beta^{-}$decay rates measured with GM counters in a usual laboratory with comfortable ambient conditions. However, in some of the experiments in section 2, temperature and air density values are plotted or given to show that there is not a drop (increase) in decay measurements with increasing (decreasing) air density. The values of air temperature and relative humidity that allow the calculation of wet air density are obtained from the meteorological station located nearby the laboratory; therefore, variations indoors are even lower than outdoors.

The GM counter tubes (GM1 and GM4) and the source are placed inside closed lead containers with up to $3 \mathrm{~cm}$ thick walls (Fig. A.1.). The lead shields reduce both the background environmental and cosmic radiation detected by the counter tube and protect from the radiation emitted by the sources. The tubes are located in the upper part of the lead shield and the mica window of the counter tube is oriented vertically downwards to receive the radiation coming from the radiation source located below, on a tray, which in turn can be placed at different distances from the window; so, the radiation beam is directed upwards. The tubes are held tight by means of a nut which must be unscrewed to loosening and eventually to replace them. This is one aspect that ensures that the distance to the source cannot be changed; another one is the plastic structure where the tray is placed: in Fig. A.1 one can see that the tray can be placed in different slots of the structure, all of which have almost no clearance. The third slot was always used for placing the tray and the source. 


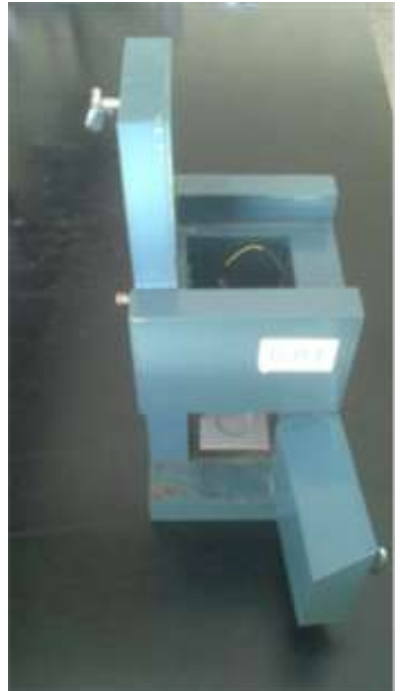

(a)

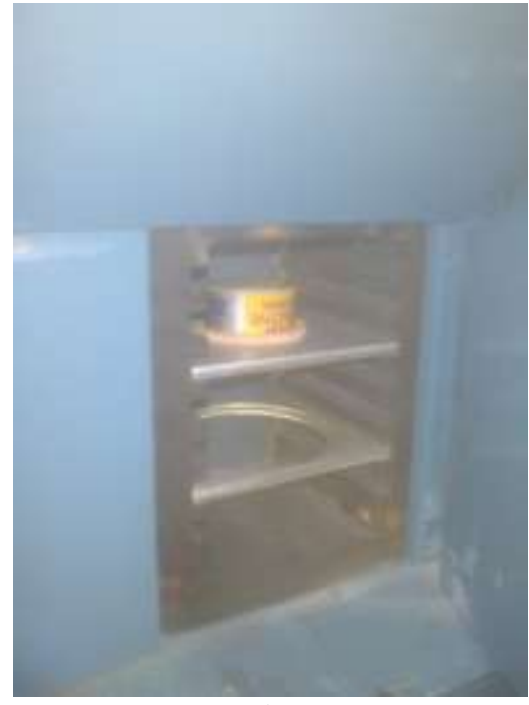

(b)

Fig. A.1. The lead container of counter tube GM1. a) The upper door used to extract the counter tube; the front door to introduce the source. b) The source inside the lead container, on a tray inserted in the third slot.

The source is always placed in the same horizontal position, Fig. A.1. First because the tray cannot move horizontally when the lead door closes and secondly, because it is fixed by means of a clamping ring (brown ring in the Figure) which in turn is fixed and located inside a notch in the tray (Fig. A.1b). Besides, the correct position of the source was always checked after moving the lead container from outside to inside the box or vice versa.

Counts detected by the GM counter tubes were registered with a scaler-timer manufactured by Ludlum, model M2200, and transferred to a computer to store and analyze the radiation counts. All potentiometers and switches in the front panel were always in the same position. Specifically, the high voltage potentiometer was always placed at $500 \mathrm{VDC}$. The connecting $50 \mathrm{ohm}$ coaxial cable between the lead container and the scaler also protects from noise. The standard cable is model L-5D2V, with a static capacity of $100 \mathrm{pF} \mathrm{m}^{-1}, 80 \mathrm{~cm}$ length, and probably polyethylene as dielectric. The capacitances of the connecting cables to GM1 and GM4 are about $73 \mathrm{pF}$ and $107 \mathrm{pF}$ respectively when measured with the LCR meter (see below meter description). These values are the same for any position and geometry of the cables (stretched out or forming a double loop). Their capacitance does not change immediately when they are close to (or even in contact with) the metal of the box. But it will be shown that, similarly to the capacitor, it changes and diminishes slowly with time (see experiments about capacitance measurements).

Scaler high voltage can be considered very stable because the power source is built with the integrated circuit LT1304: between 10 and $30^{\circ} \mathrm{C}$ the slope of the feedback voltage curve is minimal and hence the stability vs temperature is maximal. Besides, eventual small variations in voltage would not cause significant variations in recorded cpm because GM operation point is located around the middle point of the plateau.

In addition to the lead containers, a MFC was used where the containers could be placed, optionally together with the scaler. The MFC is a box of about 50x50x50 cm, made of four alternating metallic and nonmetallic materials, the latter being cork sheeting of $3 \mathrm{~mm}$ thickness and the metal being aluminum of 
$1 \mathrm{~mm}$ thickness. This metal has always been reported to produce the most noticeable effects [27, 31]. The aluminum shields are almost perfect screens for a very wide range of electromagnetic signals.

The multichannel analyzer was manufactured by Silena, model $201 \mathrm{~N}$. It basically consists in a scintillation detector which uses a $\mathrm{INa}(\mathrm{Tl})$ as the detection crystal, along with an electron multiplier zone, and provides the energy spectrum of the analyzed nuclide. According to the manufacturer, the spectrum can be drifted up to $0.5 \% /{ }^{\circ} \mathrm{C}$, but it depends on the specific tube that is being used.

\section{A.2) Low-pass RC filter and electronic components measuring equipment}

In the experiments mentioned in the Introduction section, where anomalous results are described, the question always arises on whether decay rates are uncorrelated in time with any other influence or on whether the anomalous results simply represent instrumental effects. In that context, and given that the electronics of a GM detector include a combination of resistors $\mathrm{R}$ and several capacitors C [35], a lowpass RC filter was built and tested inside the MFC to check for possible variations of its response. The low-pass filter consists of a $27.3 \mathrm{k} \Omega$ metal film resistor $\mathrm{R}$, in series with a $10 \mathrm{nF}$ nominal value multilayer ceramic capacitor C, make Epcos, model B37986N, class I (low dielectric constant), 50 VDC, with no aging effect, temperature characteristic $\mathrm{C} 0 \mathrm{G}$, temperature coefficient $\pm 30 \mathrm{ppm} / \mathrm{K}$. Both elements exhibit a very high stability over a broad temperature range.

This set up is characterized by its transfer function, given by

$$
\left|\frac{V_{o}}{V_{i}}\right|=\frac{1}{\sqrt{1+(\omega \cdot R \cdot C)^{2}}}
$$

where $\omega=2 \pi f, f$ being the applied alternating current frequency and $\tau=R C$ is the filter time constant and $V_{o}$ and $V_{i}$ are the output and input signals respectively. The ratio given by (A.1) must remain constant for a given frequency. Therefore, by registering the values of $\mathrm{V}_{\mathrm{i}}$ and $\mathrm{V}_{\mathrm{o}}$ in tests performed both inside and outside the box, it is possible to check for possible RC changes inside the metallic shield.

The RC low-pass filter input and output signals were measured with a USB oscilloscope [60]. This setup can be connected to a computer to visualize, register and analyze all the measurements.

Additional precise measurements of capacitor's impedance $Z$, reactance $X_{c}=1 / \omega C$ and dissipation factor D were performed with an HP 4263 LCR meter [45]; the test fixture HP 16089B (Kelvin clip leads) minimizes parasitics during capacitors testing. This equipment was calibrated before measurements. The meter was connected to a computer along with a temperature sensor installed in an Arduino UNO card (this sensor was always placed nearby the lead shield). The software was LABVIEW.

\section{A.3) Methods}

A.3.1) Method for the analysis of background counts, decay rates and spectrum 
To ensure that the functioning and measurements with the GM detector were reliable it was first checked with the Ra-226 source that the GM counter plateau slope was according to the specifications ( $\leq$ $0.04 \% / \mathrm{V}$ ). Secondly, by means of the $\chi^{2}$ test, it was checked that the recorded background counts (and decay rate) fitted a Poisson (or normal) distribution [35]. Later, it was also verified that background measurements were stable, and after that, a comparison between a succession of measurements both inside and outside the MFC was performed to check for possible discrepancies.

To verify the stability of the counter over time, background counts were taken (for instance), during 60 consecutive hours in periods of one minute (60 measurements per hour), and data were pooled in 60 groups of 60 data each one. These data groups were analyzed with the statistical program Statgraphics Centurion: it provides an ANOVA table and a variance check to test the equality of the means and variances respectively (of the successive groups), at the desired confidence level (95\% or 99\%). The results can be graphically represented with the multiple sample comparison option (using Tukey's honestly significant difference HSD, or the least significant difference intervals, LSD).

In long series of measurements, the data groups which exhibit the greatest differences could be also analyzed by pairs with a t-test to confirm the statistically significant difference of means and variances, (the program also provides information about all the statistics of interest). Alternatively, the differences amongst data groups can be analyzed by means of the standard error. To this end, the means (of the successive groups) can be plotted along with the error bars of $\pm 2 \sigma_{\mathrm{m}}$, where $\sigma_{\mathrm{m}}$ is the standard deviation of the mean given by $\sigma / \sqrt{ } \mathrm{N}, \sigma$ is the data standard deviation and $\mathrm{N}$ is the number of repeated counts.

Once checked the correct functioning of the GM counters, background measurements were taken inside the MFC to be compared with previous tests outside the box. For this purpose, counts were recorded in a similar way as in the outside, i.e., firstly, discontinuously during periods of several hours along several consecutive days. Afterwards, measurements were taken in a continuous way during longer periods but alternating the location of the source between outside and inside the MFC. This additional procedure was adopted to answer the question of whether some unexpected effects could be observed when measuring in this way.

To analyze and compare the results both outside and inside the MFC, different tests can be applied. So, a non-parametric test (Kruskal-Wallis) to compare medians was always performed when the requirements for an ANOVA analysis were not fulfilled.

Moreover, subsequent background counts measurements were performed from time to time aiming to check the stability of the detector and to confirm the repetition of the observed results over time, as well as to compare results in different time periods (months).

All the elements of the setup were always the same (cables, lead containers, scaler-timer and geometry of the system).

Similar procedures were followed to analyze and compare decay rates of the three aforementioned preparations both outside and inside the metallic screen. First, measurements were collected over several days during similar periods of several hours each day, in consecutive constant time intervals. The comparisons between these measurements were performed with an ANOVA test. Also sometimes, the evolution of relevant statistics was analyzed. 
Additionally to those tests, some measurements were also taken and the results analyzed when the scaler-timer was introduced inside the MFC along with the lead container to isolate (theoretically) even more the whole system from external radiations.

Later, and since in the aforementioned literature periodicities and fluctuations over long periods of time are described, it seemed reasonable to look for gradual variations (transients) in decay rates measurements when taking continuous measurements inside the MFC for lengthy periods of time (here also the consecutive one hour data groups were compared). Therefore, to complement the first tests (performed in a discontinuous way), continuous measurements were undertaken, a) keeping the preparations during about one day (or more) inside the enclosure and b), changing its location from the outside to the inside and vice versa. In this way, it was also intended (sometimes) to look for possible trends and/or coincidences, particularly with the hour of the day (as noted by Piccardi in his tests D [23] or as described by Parkhomov [61]).

The spectrum of the Cs-137 was directly obtained with the Silena MCA. The spectrum analysis was of preliminary character because the photomultiplier tube was not fully shielded with lead; therefore, the spectrum was obtained only four times outside the MFC and five times inside it. The average number of counts per channel was calculated and by this means the averaged spectrums for both outside and inside the box were obtained and compared.

\section{A.3.2) Method for measuring the low-pass RC filter time constant}

The RC low-pass filter time constant RC can be measured by means of its transfer function given by (A.1). To check for possible transfer function variations, we used the aforementioned USB digital wave form generator and oscilloscope. To this end, a sinusoidal voltage of $5 \mathrm{kHz}$ and amplitude $\mathrm{V}_{\mathrm{i}}$ of $5 \mathrm{~V}$ was fitted into the low-pass filter input; both $\mathrm{V}_{\mathrm{i}}$ and the output amplitude $\mathrm{V}_{\mathrm{o}}$ were registered by means of the same instrument.

The USB oscilloscope was set to perform one measurement of one second duration, and the input signal was scanned at a rate of $400 \mathrm{kHz}$. So, a set of 5000 measurements of both the maximum output and input voltages could be taken each second and the average was calculated. This procedure was repeated four times outside the box, and the corresponding RC average value calculated by means of the transfer function.

In a fixed position inside the box, different values of the series resistor $\mathrm{R}$ were used and the (supposedly) constant values for RC were checked.

\section{A.3.3) Method to measure the capacitance}

Measurements of impedance $\mathrm{Z}$ and capacitance $\mathrm{C}$ were performed according to the equipment's measuring procedures [45]. The selected test frequencies were $10 \mathrm{kHz}$ and $100 \mathrm{~Hz}$, therefore, parallel equivalent circuit measuring model configuration was chosen because the corresponding tests impedances were medium and high respectively; some complementary measurements at $100 \mathrm{kHz}$ were also undertaken. According to the reactance chart, the accuracies at $10 \mathrm{kHz}$ and $100 \mathrm{kHz}$ are $0.08 \%$ and $0.3 \%$ at $100 \mathrm{~Hz}$. As in all previous cases, measurements were repeated to verify the repeatability. 


\section{References}

[1 ] E. Rutherford, H. Geiger, Phil. Mag. 20, 698 (1910).

[2 ] L.F. Curtiss, J. Res. Nt. Bur. Stand. A, 4, 595 (1930).

[3 ] W. Kutzner, Z. Phys. 21, 281 (1924).

[4 ] J.L. Anderson, J. Phys. Chem., 76, No. 24, (1972).

[5 ] J.L. Anderson, G.W. Spangler, J. Phys. Chem., 77, No. 26, (1973).

[6 ] G.T. Emery, Ann. Rev. Nucl. Phys. 22, (1972) 165-202.

[7 ] K.P. Dostal, M. Nagel, D. Pabst, 1977. Z. Naturforsch. A 32A, (1977) 345-361.

[8 ] E.B. Norman, G.A. Rech, E. Browne, R.M. Larimer, M.R. Dragowsky, Y.D. Chan, M.C. P. Isaac, R.J. McDonald, A.R. Smith, Phys. Lett. B (2001).

[9 ] E. Fischbach, J.B. Buncher, J.T. Gruenwald, J.H. Jenkins, D.E. Krause , J.J. Mattes , J.R. Newport, Space Sci. Rev. 145 (2009) 285-335. DOI 10.1007/s11214-009-9518-5.

[10] J.H. Jenkins, K.R. Herminghuysen, T.E. Blue, E. Fischbach, D. Javorsek II, A.C. Kauffman, D.W. Mundy, P.A. Sturrock, J.W. Talnagi, Astrop. Phys. 37 (2012) 81-88. http://dx.doi.org/10.1016/j.astropartphys.2012.07.008.

[11] P.A.Sturrock, E. Fischbach, J. Jenkins, ApJ, 794, No1, (2014).

[12] J.H. Jenkins, E.Fischbach, D.JavorsekII, R.H.Lee, P.A.Sturrock, Appl. Rad. Isot. 74 (2013) 50-55.

[13] E.D. Falkenberg, Apeiron, 8 (2) (2001) 32-45.

[14] A.G. Parkhomov, arXiv:1010.1591v1 [physics. gen-ph]).

[15] D.J. Pons, A.D. Pons, A.J. Pons, Applied Physics Research 7 (3) (2015).

[16] S.E. Shnoll, V.A. Kolombet, E.V. Pozharskii, T.A. Zenchenko, I.M. Zvereva, A.A. Konradov, Phys. Usp. 41 (10) (1998) 1025-1035.

[17] M.V. Fedorov, L.V. Belousov, V.L. Voeikov, T.A. Zenchenko, K.I. Zenchenko, E.V. Pozharski, A.A. Konradov, S.E. Shnoll, Astrophys. Space Sci. 1 (2003) 105-112.

[18] S.E. Shnoll, Progress in Physics 2 (2) (2006) 39-45.

[19] S.E. Shnoll, Cosmophysical Factors in Stochastic Processes, American Research Press, Rehoboth (NM, USA), 2012.

[20] V.A. Namiot, S.E. Shnoll, Phys. Lett. A 359 (2006) 249-251.

[21] D. Rabounski, L. Borissova, Progress in Physics 10 (2) (2014) 63-70.

[22] T.A. Zenchenko, A.A. Konradov, K.I. Zenchenko, Biophotonics and Coherent Systems in Biology (2007) 225-233.

[23] Geo-cosmic relations; the earth and its macro-environment. Proceedings of the First International Congress on Geo-cosmic Relations. Pudoc, Wageningen, 1990.

[24] International Crimean Conference, Cosmos and Biosphere (2003). http://www.science-center.net.

[25] International Symposium. Relations of Biological and Physicochemical Processes with Solar Activity and other Environmental Factors, Pushchino, 1993.

[26] M.N. Zhadin, Bioelectromagnetics 45 (2001) 22-27.

[27] G. Piccardi, The Chemical Basis of Medical Climatology, Charles C. Thomas, Sprinfield, IL, 1962.

[28] J.H. Nelson, RCA Review, Radio and a Res. Eng. March (1951).

[29] B.M. Vladimirsky, A.V. Bruns, Izvestiya - Atmospheric and Oceanic Physics 46 (8) (2010) 935-951.

[30] A. Giao, Rend. Accad. Naz. Lincei, 25 (8) (1958).

[31] W. Reich, Selected Writings: An Introduction to Orgonomy, Farrar, Straus \& Giroux, NY, (1960).

[32] S. Müschenich, R. Gebauer, The (Psycho-)Physiological Effects of the Reich Orgone Accumulator, Dissertation, University of Marburg, West Germany, 1985.

[33] J. DeMeo, WATER Journal 3, (2011) 1-47. http://www.waterjournal.org/.

[34] N. Farashchuk, M. Fomitchev-Zamilov, Y. Tsyuman. WATER Journal 6, (2014) 26-34. http://www.waterjournal.org/. 
[35] G.F. Knoll, Radiation Detection and Measurement, third ed., John Willey \& Sons, New York, 1999.

[36] H. Levene, In Contributions to Probability and Statistics: Essays in Honor of Harold Hotelling, I. Olkin et al. eds., Stanford University Press, (1960) 278-292.

[37] M. Kimura, Phys. Rev. 80 (1950), 761.

[38] J.E.Turner, Atoms, Radiation and Radiation Protection, Wiley-VCH Verlag GmbH\&Co. KGaA, Weinheim (2004).

[39] P.L. Campion, Nucl. Instrum. Meth. 112 (1973) 75.

[40] E. Fenyves, O. Haiman, The Physical Principles of Nuclear Radiation Measurements, Academic Press, New York and London 1969.

[41] H. Genz, D.S. Harmer, R.W. Fink, Nucl. Instrum. Meth. 60, (1968) 195.

[42] L.A. Facorro Ruiz, Curso de termodinámica, ediciones Melior, Buenos Aires, Argentina,1974.

[43] R.S. Davis, Metrologia 18 (3) (1982) 171.

[44] A. Picard, R.S. Davis, M. Glaser, K. Fujii, Metrologia 45 (2) (2008) 149.

[45] Magnetic Storms Online http://www.tesis.lebedev.ru/en/magnetic_storms.html?m=7\&d=26\&y=2014.

[46] A. Tanarro Sanz, Instrumentación Nuclear, Junta de Energía Nuclear, Madrid 1970.

[47] http://bwrcs.eecs.berkeley.edu/Classes/icdesign/ee141 f01/Notes/chapter4.pdf.

[48] Agilent Impedance Measurement Handbook 4th Edition.

[49] G. Choppin, J.O. Liljenzin, J. Rydberg, Radiochemistry and Nuclear Chemistry, Third Edition, ISBN-13: 978-0-7506-7463-8, ISBN-10: 0-7506-7463-6.

[50] R. Coelho, Physics of Dielectrics for the Engineer, Elsevier Scientific Publishing Co. Amsterdam, Oxford, New York, 1979.

[51] C.M. Lederer, J.M. Hollander, I. Perlman, Table of Isotopes, Sixth Edition, John Wiley and Sons, Inc. New York, London, Sidney, 1967.

[52] K.S. Krane, Introductory Nuclear Physics, second ed. John Wiley and Sons, 1988.

[53] V. Chisté, M.M. Bé, C. Dulieu, DOI: 10.1051/ndata:07122. http://nd2007.edpsciences.org or http://dx.doi.org/10.1051/ndata:07122.

[54] P. Christmas, Nucl. Phys. 55 (1964) 577-592.

[55] http://www.nucleide.org/DDEP_WG/Nuclides/Tl-204_tables.pdf.

[56] Centronic. Geiger Selection Guide, http://www.centronic.co.uk/downloads/Geiger-Selection-Guide.pdf.

[57] www.centronic.co.uk_downloads_Geiger-Selection-Guide.pdf.

[58] J.E. Jenkins, D.W. Mundy E. Fischbach, Nucl. Instr. and Meth. A 620 (2010) 332-342.

[59] C.G. Found, Ionization potentials of argon, nitrogen, carbon monoxide, helium, hydrogen and mercury and iodine vapors, Physical Review 16 (1920) 41.

[60] www.digilentinc.com.

[61] A.G. Parkhomov, Journal of Modern Physics, 2 (2011) 1310-1317. 